\title{
Type III TGF- $\beta$ receptor promotes FGF2-mediated neuronal differentiation in neuroblastoma
}

\author{
Erik H. Knelson, ${ }^{1,2}$ Angela L. Gaviglio, ${ }^{1}$ Alok K. Tewari, ${ }^{1,2}$ Michael B. Armstrong, ${ }^{3}$ \\ Karthikeyan Mythreye, ${ }^{4}$ and Gerard C. Blobe ${ }^{1,4}$ \\ 1Department of Pharmacology and Cancer Biology, ${ }^{2}$ Medical Scientist Training Program, ${ }^{3}$ Department of Pediatrics, and ${ }^{4}$ Department of Medicine, \\ Duke University Medical Center, Durham, North Carolina, USA.
}

\begin{abstract}
Growth factors and their receptors coordinate neuronal differentiation during development, yet their roles in the pediatric tumor neuroblastoma remain unclear. Comparison of mRNA from benign neuroblastic tumors and neuroblastomas revealed that expression of the type III TGF- $\beta$ receptor (TGFBR3) decreases with advancing stage of neuroblastoma and this loss correlates with a poorer prognosis. Patients with MYCN oncogene amplification and low TGFBR3 expression were more likely to have an adverse outcome. In vitro, T $\beta$ RIII expression was epigenetically suppressed by MYCN-mediated recruitment of histone deacetylases to regions of the TGFBR 3 promoter. T $\beta$ RIII bound FGF2 and exogenous FGFR1, which promoted neuronal differentiation of neuroblastoma cells. T $\beta$ RIII and FGF2 cooperated to induce expression of the transcription factor inhibitor of DNA binding 1 via Erk MAPK. T $\beta$ RIII-mediated neuronal differentiation suppressed cell proliferation in vitro as well as tumor growth and metastasis in vivo. These studies characterize a coreceptor function for T $\beta$ RIII in FGF2-mediated neuronal differentiation, while identifying potential therapeutic targets and clinical biomarkers for neuroblastoma.
\end{abstract}

\section{Introduction}

Neuroblastoma (NB), the most common cancer in infancy (1), arises from developing neurons in the sympathetic ganglia or adrenal gland. While early-stage tumors are treated effectively and may regress spontaneously, survival in patients with advanced-stage tumors is below $40 \%(2,3)$. Clinical heterogeneity and treatment morbidity $(4,5)$ have driven the development of genetic and molecular screening approaches to identify children who may be spared intensive therapy (6-8).

MYCN oncogene amplification occurs in $20 \%$ of NB cases and portends a poor prognosis $(7,9,10)$. MYCN epigenetically activates and represses target genes to promote NB cell proliferation and forestall neuroblast differentiation (11). While MYCN-targeted therapies have proven disappointing, the oncogene's pleiotropic actions have generated interest in manipulating downstream transcriptional targets, either directly or by inhibiting the epigenetic effects of MYCN, including the recruitment of histone deacetylases (HDACs) (12).

Neuroblast differentiation represents a validated treatment strategy in NB. Retinoic acid is used clinically to target residual tumor cells by promoting neuronal differentiation (13). In vitro studies with retinoic acid and other differentiating agents have generated useful model systems for the study of neuroblast differentiation, but no additional therapies have emerged (14). While

Authorship note: Karthikeyan Mythreye and Gerard C. Blobe contributed equally to this work.

Conflict of interest: The authors have declared that no conflict of interest exists.

Note regarding evaluation of this manuscript: Manuscripts authored by scientists associated with Duke University, The University of North Carolina at Chapel Hill, Duke-NUS, and the Sanford-Burnham Medical Research Institute are handled not by members of the editorial board but rather by the science editors, who consult with selected external editors and reviewers.

Citation for this article: J Clin Invest. 2013;123(11):4786-4798. doi:10.1172/JCI69657. the growth factor pathways involved in neuroblast differentiation in development are well described (15), the precise roles of these pathways in NB remain unclear.

Previous studies suggest that TGF- $\beta$ superfamily signaling is disrupted in NB (16-19). Decreased expression of the type III TGF- $\beta$ receptor $(T G F B R 3)$ has been reported in advanced-stage NB $(16,20)$. TGFBR3 was also identified in the top 20 genes most decreased in NB compared with human fetal neuroblasts (21). T $\beta$ RIII binds ligands that are known to promote neuronal differentiation of neuroblasts (22-26), but the function of T $\beta$ RIII in NB is unknown.

FGFs have important roles in neuronal development (27), yet their role in NB has not been explored. FGF2 has been shown to promote neuronal differentiation of neural-crest tumor cells via the Erk MAPK pathway $(26,28-30)$. Erk signaling is also critical to retinoic acid- and $\alpha$-lipoic acid-induced neuroblast differentiation $(31,32)$, suggesting a broader involvement for this pathway in NB differentiation.

T $\beta$ RIII is able to bind FGF2 via glycosaminoglycan (GAG) modifications (33), which form ternary complexes with FGFs and FGF receptors in neuronal development (27). T $\beta$ RIII has been shown to modulate FGF2 signaling in cardiomyocytes (34). However, the effects of T $\beta$ RIII on FGF signaling and biology in NB have not been explored. Here, we investigate the role of T $\beta$ RIII in NB pathogenesis, uncovering novel clinically relevant roles in FGF signaling and FGF-mediated biology.

\section{Results}

T $\beta$ RIII expression is decreased in NB. T $\beta$ RIII expression is decreased in many cancers, with T $\beta$ RIII functioning to suppress tumor growth and metastasis (35). Previous reports suggest a decrease in T $\beta$ RIII expression in NB $(16,20,21)$. To explore a potential role for T $\beta$ RIII in NB, we determined mRNA expression in a normalized microarray 
A
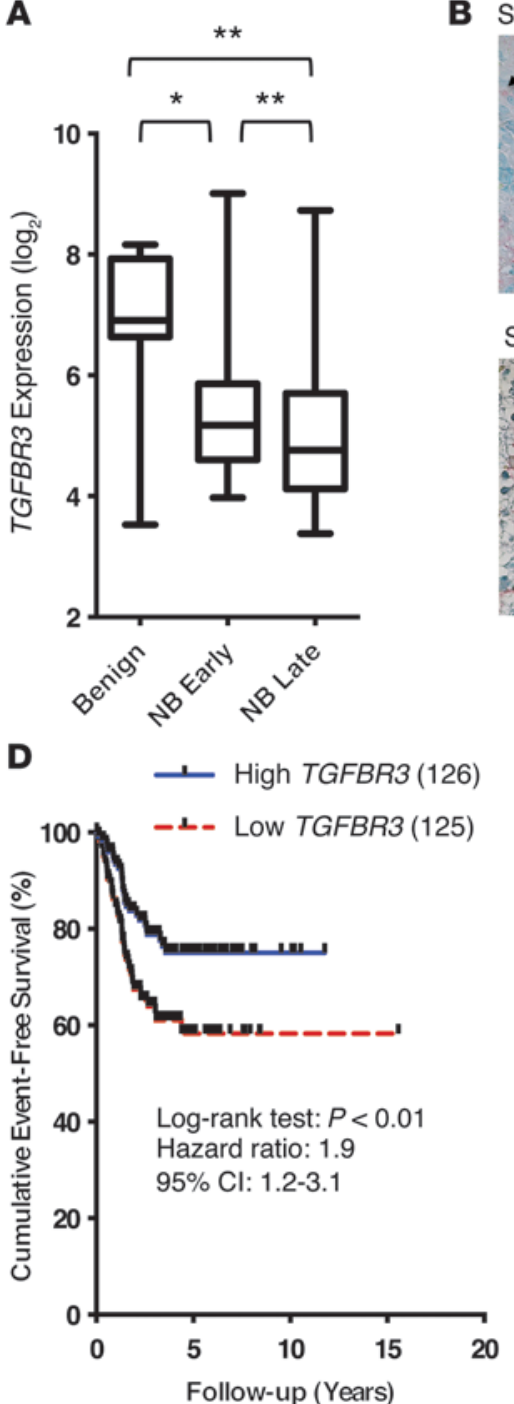

B

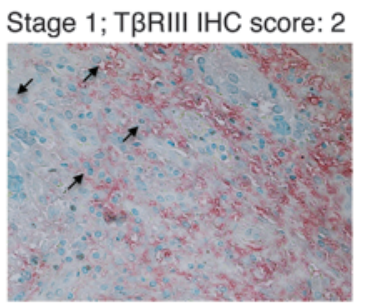

Stage 2; TBRIII IHC score: 1
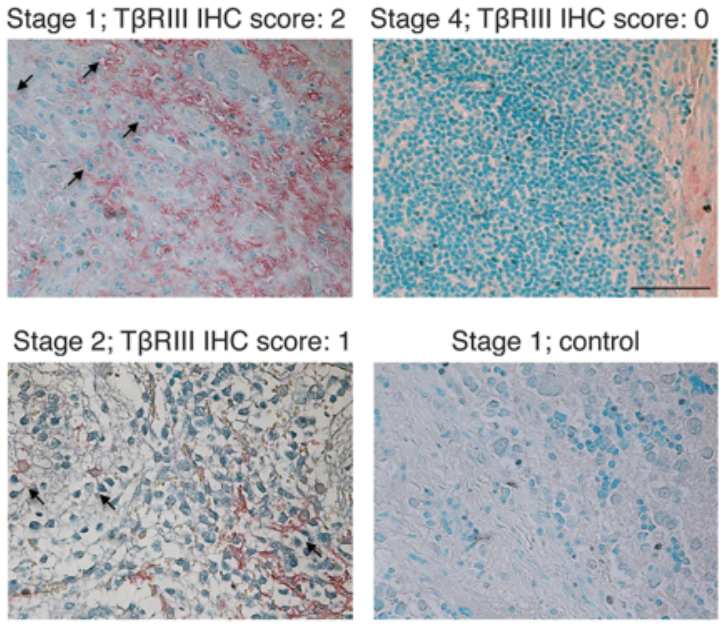

Stage 1; control
C

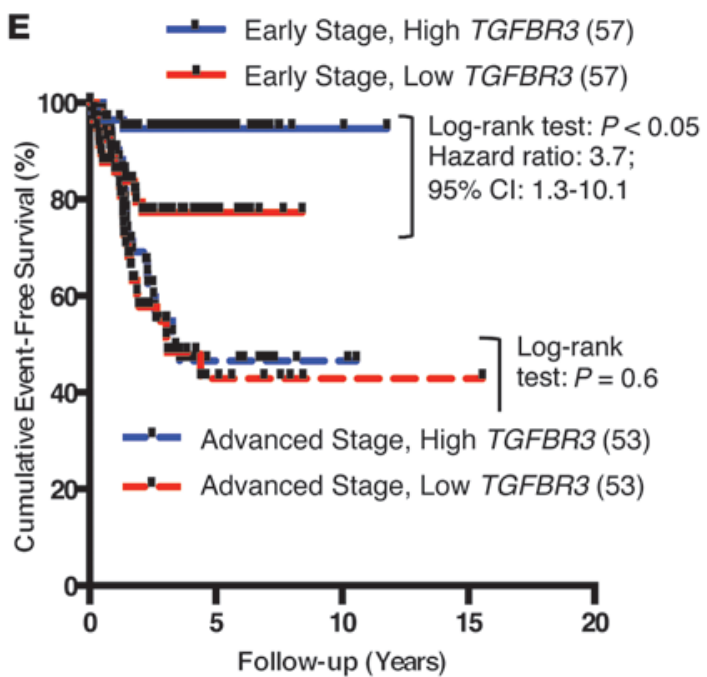

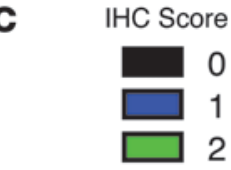

NB Early NB Late

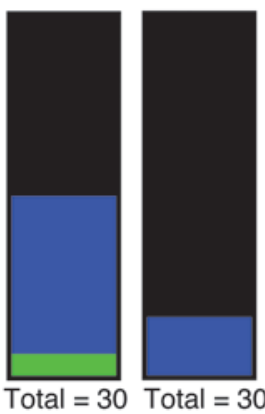

$\mathbf{F}$

I $^{125}$ TGF- $\beta 1$ Binding, T $\beta R I I I$ pull-down

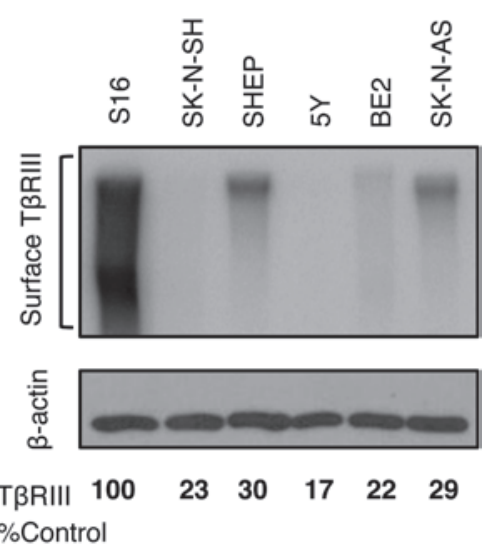

Figure 1

T $\beta$ RIII expression is decreased in NB. (A) TGFBR3 expression in the microarray data set. Data are presented as median (horizontal bars) and interquartile range (boxes). $P<0.001$, Kruskal-Wallis. ${ }^{*} P<0.05,{ }^{*} P<0.01$, intergroup comparisons (Mann-Whitney). $n=11$ benign neuroblastic tumors (ganglioneuroma/ganglioneuroblastoma); $n=79 \mathrm{NB}$ early-stage tumors (INSS stage 1/2); $n=123$ NB late-stage tumors (INSS stage 3/4). (B) Immunohistochemistry (IHC) of NB tumor samples using a T $\beta R$ III antibody and prebleed control serum. Original magnification, $\times 20$; scale bar: $50 \mu \mathrm{M}$. Arrows point to cell-associated staining. (C) Quantification of immunohistochemistry by stage of disease (30 early, 30 late). $P<0.01$ for mean immunohistochemistry score (Mann-Whitney). (D) Event-free survival in NB with low (bottom 50\%; red) and high (top 50\%; blue) TGFBR3 expression in the Oberthuer data set (36). (E) Event-free survival split by stage of disease. (F) I25 TGF- $\beta$ binding and crosslinking with T $\beta$ RIII pull-down in NB cell lines compared with S16 Schwann cell line. ( $\mathbf{D}$ and $\mathbf{E}$ ) Numbers in parentheses indicate the number of samples. Background and $\beta$-actin-normalized integrated density for T $\beta$ RIII are shown as percent control.

data set $(n=213$; Figure $1 \mathrm{~A})$. Compared with that in benign neuroblastic tumors, TGFBR3 mRNA expression was decreased in NB, with an additional significant decrease in advanced-stage NB compared with early-stage disease (Figure 1A). We performed T $\beta$ RIII immunohistochemistry in 60 primary tumor samples (Figure 1B), demonstrating a decrease in T $\beta R I I I$ protein expression in advancedstage tumors (Figure 1C).

As decreased TRRIII expression is a frequent event in NB, we sought to determine the prognostic significance of T $\beta$ RIII expression using publicly available data sets $(36,37)$. Low T $\beta R$ RII expression was significantly associated with decreased event-free survival (Figure 1D and
Supplemental Figure 1A; supplemental material available online with this article; doi:10.1172/JCI69657DS1). T $\beta R I I I$ expression further stratified patients with early-stage disease (Figure 1E and Supplemental Figure 1B), selecting a subpopulation with high T $\beta$ RIII expression and an excellent prognosis. Based on these data, we proceeded to identify model systems for further study of the role of T $\beta R$ RII in NB. Compared with the neural crest-derived S16 Schwann cell line, NB cell lines had relatively low T $\beta R I I I$ expression (Figure 1F). In the context of NB cells, the SHEP and SK-N-AS cell lines had intermediate levels of T $\beta$ RIII expression, while the 5Y, SK-N-SH, and BE2 cell lines had the lowest T $\beta$ RIII expression (Figure 1F). 


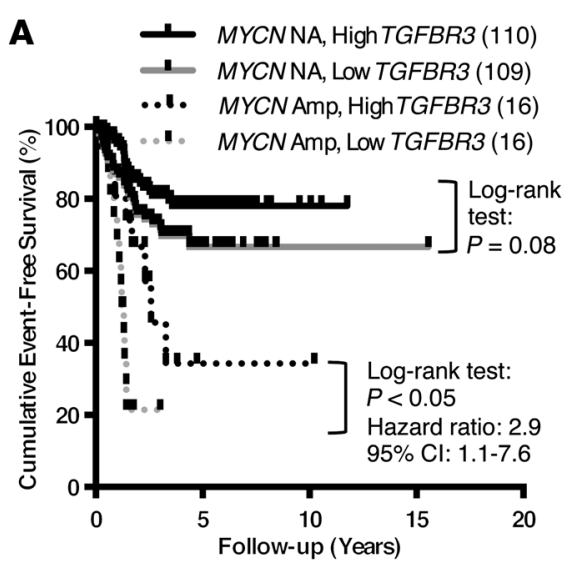

E SHEP-21N MYCN Tet-repression

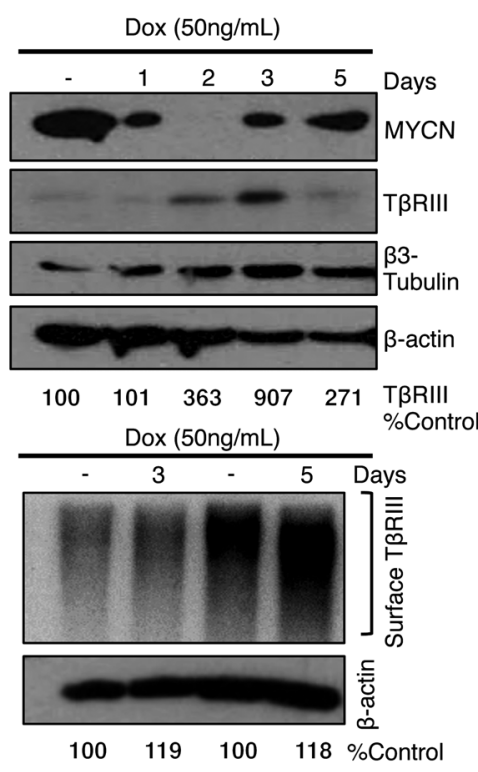

B

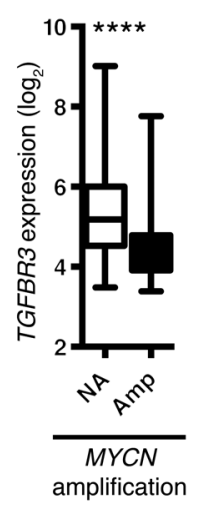

C

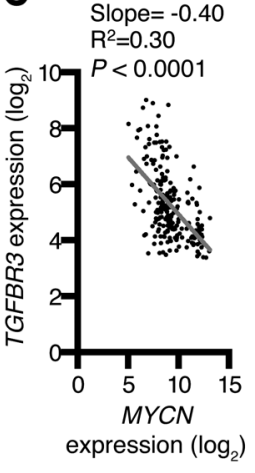

D

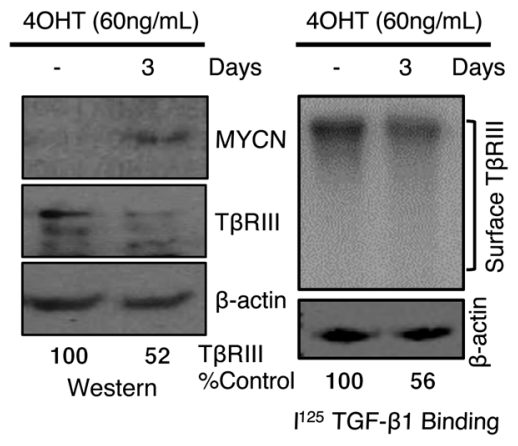

$\mathbf{F}$
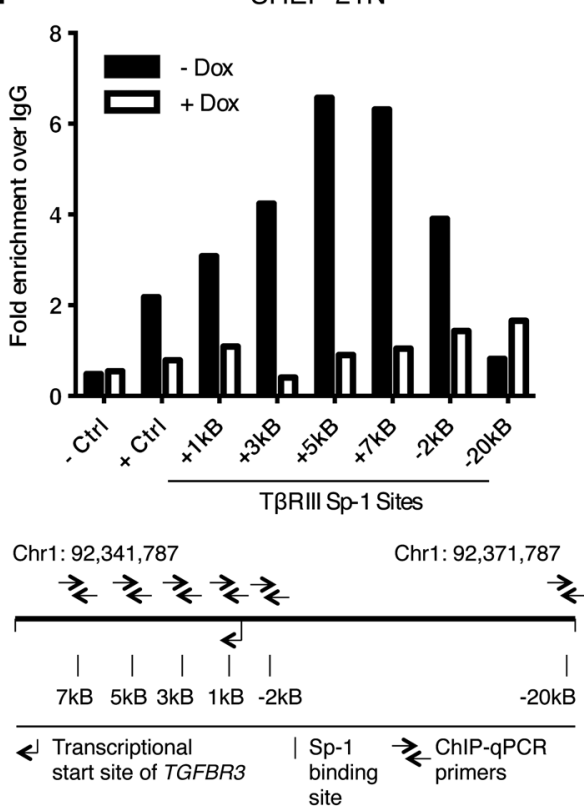

G

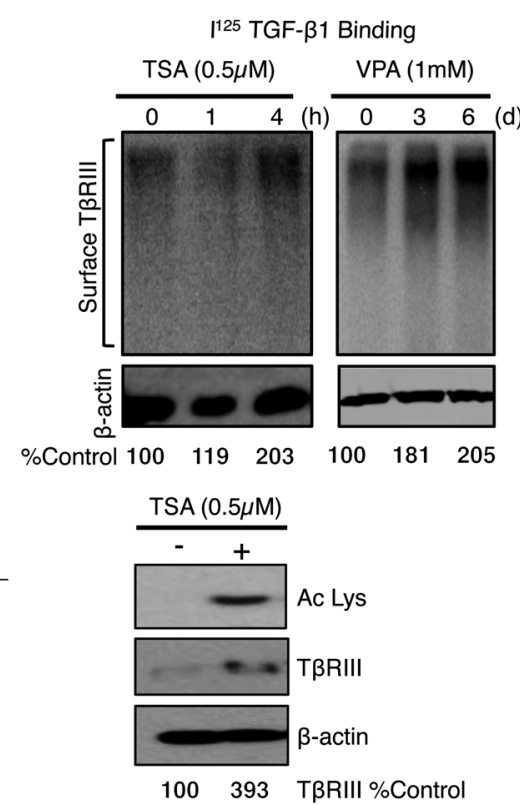

Figure 2

MYCN suppresses T $\beta$ RIII expression. (A) Analysis of event-free survival split by MYCN amplification status in NB with low (bottom $50 \%$; gray) and high (top 50\%; black) TGFBR3 expression in the Oberthuer data set (36). Amp, MYCN amplified (dashed lines); NA, nonamplified (solid lines). Numbers in parentheses indicate the number of samples. (B) Microarray data set analysis for TGFBR3 expression. Data are presented as median (horizontal bars) and interquartile range (boxes). ${ }^{* * *} P<0.0001$ (Mann-Whitney). (C) Linear regression of $M Y C N$ and TGFBR3 expression in the microarray data set. (D) Western blot and I25 TGF- $\beta$ binding and crosslinking with T $\beta$ RIII pull-down of SK-N-AS-MYCNERinducible cell line in the presence and absence of 4-hydroxytamoxifen (4OHT) to stabilize MYCN. (E) SHEP-21N-repressible cell line in the presence and absence of doxycycline (Dox) to repress MYCN expression. Dox was replenished at day 3 for the 5-day treatment in the binding experiment. (F) ChIP in SHEP-21N cells using primers for Sp-1 binding sites in T $\beta$ RIII. Data are representative of 3 experimental replicates with similar trends. (G) I 125 TGF- $\beta$ binding and crosslinking with T $\beta$ RIII pull-down in the presence and absence of trichostatin A (TSA) (1- and 4-hour treatments) and valproic acid (VPA) (3- and 6-day treatments) at the concentrations shown. Western blots for acetyl-lysine (Ac Lys) and T $\beta$ RIII in the presence and absence of trichostatin A (4-hour treatment). Background and $\beta$-actin-normalized integrated density for T $\beta$ RIII are shown as percent control.

MYCN suppresses T $\beta$ RIII expression. MYCN oncogene amplification occurs in a subset of patients with NB and confers a poor prognosis (ref. 38 and Figure 2A). Previous work by Iolascon et al. suggested a correlation between MYCN amplification and T $\beta$ RIII protein expression (16). A survival analysis showed that patients with MYCN amplification and low T $\beta$ RIII expression had the worst prognosis (Figure 2A and Supplemental Figure 1B). In our meta-analysis of microarray data sets, T $\beta$ RIII expression was decreased in NB with MYCN amplification (Figure 2B). Consistent with this decrease, TGFBR3 mRNA expression inversely correlated with MYCN mRNA expression (Figure 2C).

To investigate whether MYCN suppresses T $\beta$ RIII expression in NB cells, we used complementary inducible and repressible cell systems (39). MYCN induction decreased T $\beta$ RIII expression (Figure 2D), while MYCN repression increased T $\beta$ RIII expression (Figure 2E). Further, as doxycycline-mediated repression of MYCN waned, 
T $\beta$ RIII suppression returned (Figure $2 \mathrm{E}$ ). Interestingly, expression of the neuronal differentiation marker $\beta 3$-tubulin paralleled the rise of T $\beta$ RIII expression, suggesting that neuronal differentiation may be linked to MYCN-suppressed T $\beta$ RIII expression. Together, these data demonstrate that T $\beta$ RIII expression is suppressed by MYCN in NB tumors and cell lines.

MYCN represses gene transcription via recruitment of HDACs and DNA methyltransferases, specifically at sites of Miz1/Sp-1 transcription (11). Since T $\beta$ RIII has $4 \mathrm{Sp}-1$ binding sites in its promoter region and 2 more downstream of the transcriptional start site, we hypothesized that MYCN suppresses T $\beta$ RIII expression via epigenetic silencing. ChIP demonstrated a direct interaction between MYCN and the $4 \mathrm{Sp}-1$ sites in the promoter region of T $\beta$ RIII (Figure 2F). MYCN also directly bound an Sp- 1 site $2 \mathrm{kB}$ downstream of the transcriptional start site but failed to bind an Sp- 1 site $20 \mathrm{kB}$ downstream as well as a negative control site $90 \mathrm{kB}$ downstream (Figure $2 \mathrm{~F})$. Furthermore, treatment of $M Y C N$-amplified NB cells with the HDAC inhibitors, trichostatin A and valproic acid, increased T $\beta$ RIII expression (Figure $2 \mathrm{G}$ ), suggesting that T $\beta \mathrm{RIII}$ expression could be rescued from MYCN/HDAC-mediated epigenetic silencing.

$T \beta R I I I$ promotes neuronal differentiation. Since T $\beta$ RIII expression paralleled expression of a differentiation marker (Figure 2E) and T $\beta$ RIII binds known differentiating ligands (22-26), we sought to determine whether T $\beta$ RIII expression promotes neurite outgrowth and expression of differentiation marker proteins in NB cells. Neurite outgrowth was enhanced in cells with increased T $\beta$ RIII expression (Figure 3, A and B, and Supplemental Figure $2, \mathrm{~A}, \mathrm{C}$, and D) and decreased in cells with T $\beta$ RIII knockdown (Figure 3C and Supplemental Figure 2, A and D). Similarly, biochemical markers of neuronal differentiation were increased in NB cells with increased T $\beta$ RIII expression (Figure 3, D and E) and decreased in cells with T $\beta$ RIII knockdown (Figure 3, F and G, and Supplemental Figure 2A). This decrease was rescued by restoring T $\beta$ RIII expression (Figure 3, F and G). These results demonstrate that T $\beta$ RIII promotes neuronal differentiation of NB cells. In our meta-analysis of microarray data sets, TGFBR3 expression correlated with expression of the differentiation marker and neuronal development regulator SOX10 (Figure $3 \mathrm{H}$ and refs. 40, 41).

$T \beta R I I I$ promotes neuronal differentiation via FGF2 signaling. To determine whether T $\beta$ RIII promotes neuronal differentiation by enhancing the effects of its ligand binding partners, we treated NB cells with ligands previously shown to promote neuronal differentiation: TGF- $\beta 1$, BMP2, and FGF2 (Supplemental Figure 3A). TGF- $\beta 1$ did not enhance differentiation and BMP2 induced differentiation in only a subset of NB cells (Supplemental Figure 3A). Further, increasing T $\beta$ RIII expression failed to alter canonical Smad phosphorylation in response to TGF- $\beta 1$ or BMP2 (Supplemental Figure 3C), while treatment with inhibitors of TGF- $\beta$ and BMP signaling failed to attenuate the differentiating effects of T $\beta$ RIII (Supplemental Figure $3 \mathrm{D})$. These results suggested that the effects of T $\beta$ RIII were not mediated by TGF- $\beta 1$ or BMP2. In contrast, FGF2 treatment induced differentiation in all NB cell lines; this effect was enhanced by high T $\beta$ RIII expression and abrogated by T $\beta$ RIII knockdown (Figure 4, A, C, and D, and Supplemental Figure 3A).

T $\beta$ RIII is known to bind FGF2 via GAG chains (33). Consistent with a role for T $\beta$ RIII in mediating differentiation via FGF2, the extracellular domain and its GAG chains were required for neuronal differentiation in both gain- and loss-of-function contexts in multiple cell lines (Figure 4, B and C; Supplemental Figure 3, E and F; and Supplemental Figure 4, A and B). Furthermore, T $\beta$ RIII sig- nificantly enhanced the differentiating effects of low-dose FGF2 in a GAG-dependent manner (Figure 4C). These results demonstrate that GAG chains on T $\beta$ RIII promote neuronal differentiation and enhance the differentiating effects of FGF2 treatment.

Since T $\beta$ RIII enhanced FGF2-mediated neuronal differentiation, we investigated whether T $\beta$ RIII acts as an FGF coreceptor in NB cells. Consistent with a coreceptor role, T $\beta$ RIII specifically bound FGF2 and enhanced FGF2 surface binding via GAG chains (Figure 4D and Supplemental Figure 4, C and D). Since heparan sulfate chains on cell surface receptors can bind both FGF ligands and receptors in neurons (27), we investigated whether T $\beta$ RIII could interact with GAG attachment sites on FGF receptors. Indeed, exogenous T $\beta$ RIII coimmunoprecipitated exogenous FGFR1 in a GAG-dependent manner (Figure 4E and Supplemental Figure 4E). Furthermore, endogenous T $\beta$ RIII coimmunoprecipitated exogenous FGFR1; this interaction was abrogated by T $\beta$ RIII knockdown (Supplemental Figure 4E). We also observed an interaction among endogenous proteins that increased with FGF2 treatment (Supplemental Figure 4E). Treatment with an FGF2 inhibitory antibody failed to abrogate the differentiating effects of T $\beta$ RIII (Supplemental Figure 3B), supporting the potential for a ligand-independent receptor crosstalk mechanism in addition to the potentiation of ligand effects by T $\beta$ RIII. These results support a functional interaction among T $\beta$ RIII, FGF2 ligand, and FGFR1 in NB cells.

$T \beta R I I I$ enhances FGF2 signaling to promote neuronal differentiation. Consistent with a coreceptor role, T $\beta$ RIII enhanced both shortterm (minutes to hours) and long-term (days) FGF2-mediated Erk phosphorylation in a GAG-dependent manner (Figure 5A and Supplemental Figure 5A). Silencing of T $\beta$ RIII expression decreased basal Erk phosphorylation and blunted the response to FGF2 treatment (Figure 5A). To investigate the contribution of FGF signaling pathways to T $\beta$ RIII/FGF2-induced neuronal differentiation, we blocked FGF receptor kinase activity with pharmacologic inhibitors (PD-173074, SU-5402) or a dominant-negative FGFR1 construct (ref. 42; Figure 5, B and C; and Supplemental Figure 5, B and D). In all cases, inhibition of FGF receptor tyrosine kinase function attenuated the differentiating effects of T $\beta$ RIII expression in the presence and absence of exogenous FGF2. Similarly, pharmacologic inhibition of downstream MEK/Erk MAPK signaling with U0126 and CI-1030 attenuated the differentiating effects of T $\beta$ RIII expression in the presence and absence of ligand (Figure 5B and Supplemental Figure 5, C and D). These results demonstrate that T $\beta$ RIII and its GAG chains promote neuronal differentiation and enhance FGF2-induced differentiation in NB cells via FGF receptors and downstream Erk MAPK signaling.

$T \beta R I I I$ and FGF2 cooperate to induce Id1 expression. Similar to previous work demonstrating that FGF2 promotes differentiation of neural crest-derived cells via Erk MAPK and the transcription factor inhibitor of DNA binding 1 (Id1) (30), we found that FGF2 induced Id 1 protein expression in NB cells within 1 hour of treatment, followed by a gradual decrease in expression (Figure 6A). Interestingly, T $\beta$ RIII knockdown completely abrogated FGF2induced Id 1 expression. We also observed increases in Id 1 protein levels in response to FGF2 over the longer time course of neuronal differentiation; this increase was inhibited by T $\beta$ RIII knockdown and could be rescued by restoring T $\beta$ RIII expression with GAG modifications (Figure 6B). Likewise, basal Id1 expression and FGF2-induced increases in Id 1 expression were enhanced by T $\beta$ RIII overexpression in a GAG-dependent manner (Supplemental Figure 5E). T $\beta$ RIII- and FGF2-induced Id1 expression changes were abro- 
A

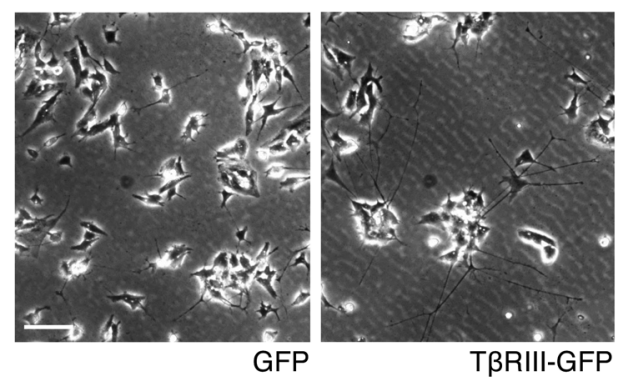

D $5 Y$

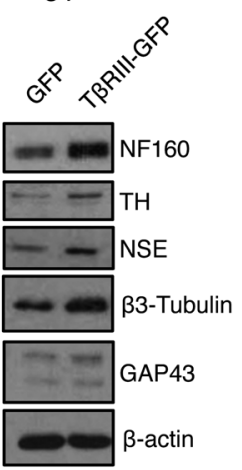

100217 NF160 \%Control

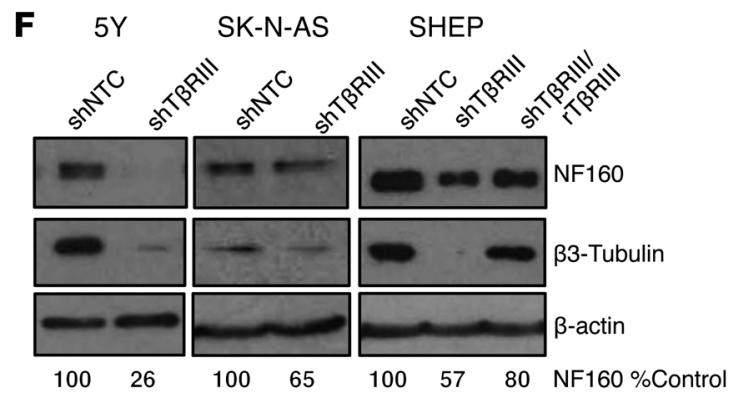

B

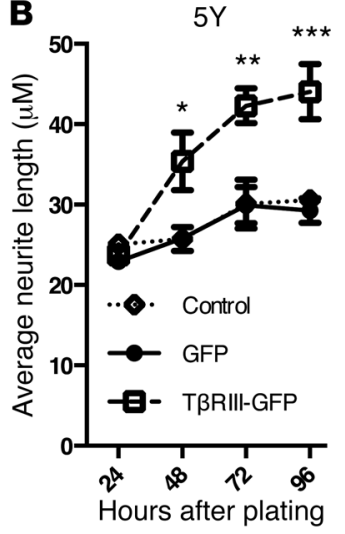

C

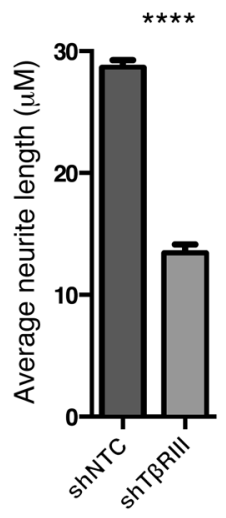

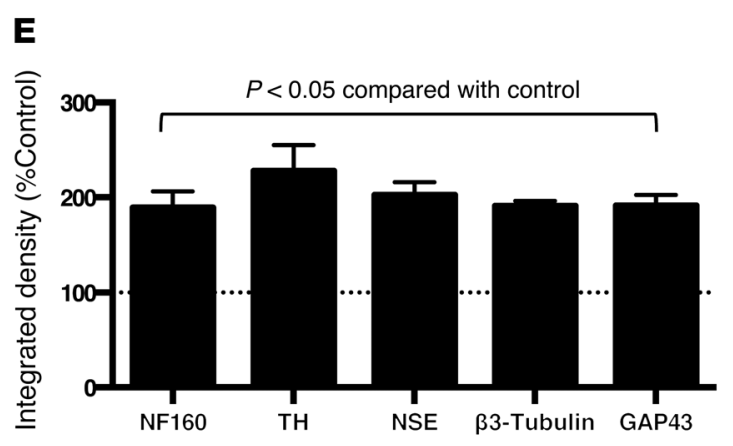

G

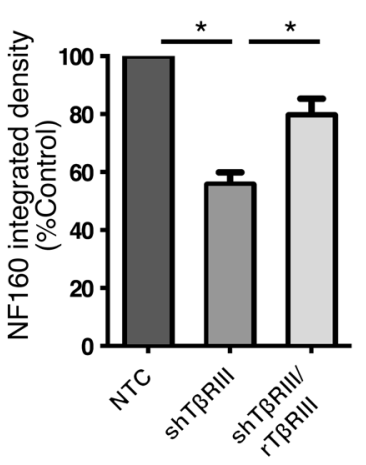

Figure 3

T $\beta$ RIII promotes neuronal differentiation of NB cells. Transient transductions with T $\beta R$ IIII-GFP, GFP control, nontargeted control shRNA (shNTC),

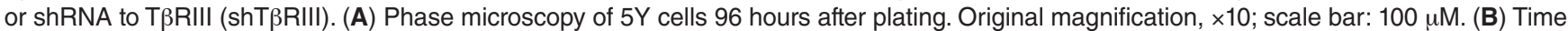
course of $5 Y$ cell neurite length (mean of 3 fields \pm SEM). Adenoviral transduction at 24 hours. $P<0.0001$ for main effects of time and receptor expression (2-way ANOVA); interaction $P<0.05$; ${ }^{*} P<0.05$, ${ }^{* \star} P<0.01$, ${ }^{\star * \star} P<0.001$ (Bonferroni post-hoc comparisons shown for T $\beta R$ IIII-GFP compared to GFP and control). (C) $5 \mathrm{Y}$ cell neurite length (mean of 3 fields \pm SEM) after 96 hours of TRRIII knockdown. ${ }^{* * \star *} P<0.0001$ (2-tailed Student's $t$ test). (D) Western blot for neurofilament $160 \mathrm{kDa}$ (NF160), tyrosine hydroxylase (TH), neuron-specific enolase (NSE), $\beta 3$-tubulin, and GAP43 after 96-hour transduction. Densitometry for NF160 normalized to $\beta$-actin is shown as percent control. (E) Quantification of differentiation markers from 3 independent experiments in $5 \mathrm{Y}$ cells normalized to $\beta$-actin (mean increase above control $\pm \mathrm{SEM}$ ). $P<0.05$ for all markers (1-sample Student's $t$ test). (F) Differentiation markers after 72-hour T $\beta R$ IIII knockdown and rescue with knockdown-resistant rat T $\beta R$ IIII ( $r T \beta R$ III). Densitometry for NF160 normalized to $\beta$-actin is shown as percent control. (G) Quantification of NF160 from 3 independent experiments (mean \pm SEM) in SHEP cells normalized to $\beta$-actin. ${ }^{*} P<0.05$ (1-sample $t$ test and 2-tailed Student's $t$ test). (H) Microarray data set expression of SOX10 in tumors with low (bottom 10\%) and high (top 10\%) TGFBR3 expression (median [horizontal bars] and interquartile range [boxes]). ${ }^{* \star *} P<0.001$ (Mann-Whitney).

gated by treatment with FGFR and Erk MAPK inhibitors (Figure 6C). Consistent with a downstream role for Id 1 in the differentiation pathway, Id 1 knockdown attenuated the differentiating effects of T $\beta$ RIII expression in the presence of FGF2 treatment (Figure 6D). Moreover, in specimens from patients with NB, ID1 mRNA expression positively correlated with TGFBR3 mRNA expression (Figure 6E). These results demonstrate that T $\beta$ RIII and FGF2 cooperate to induce Id 1 expression. Furthermore, Id 1 expression is critical to the differentiating effects of T $\beta$ RIII/FGF2 and correlates with T $\beta$ RIII expression in specimens from patients with NB. 
$\mathbf{A}$

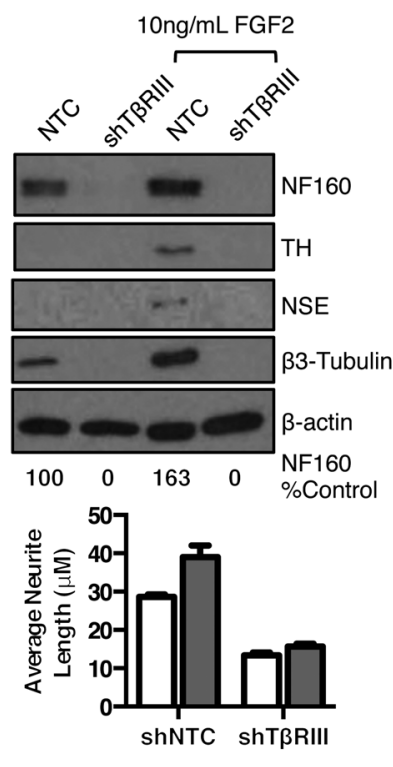

B

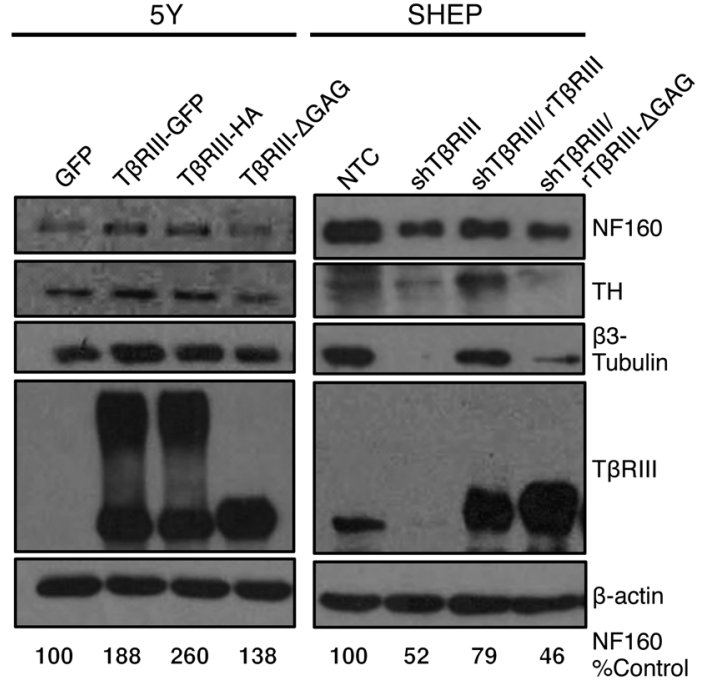

C
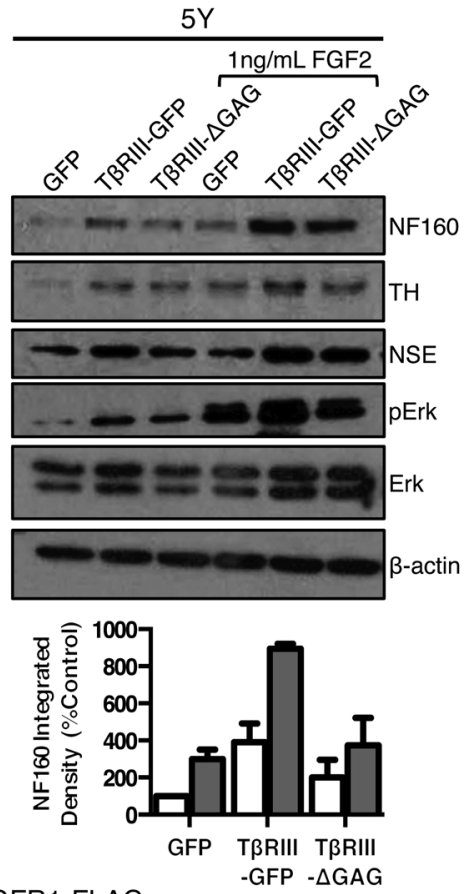

$\mathbf{E}$

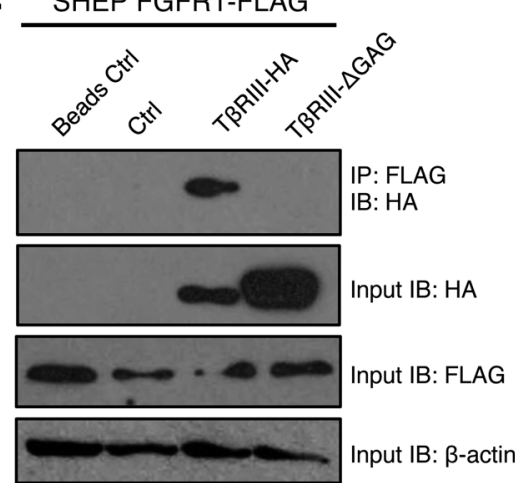

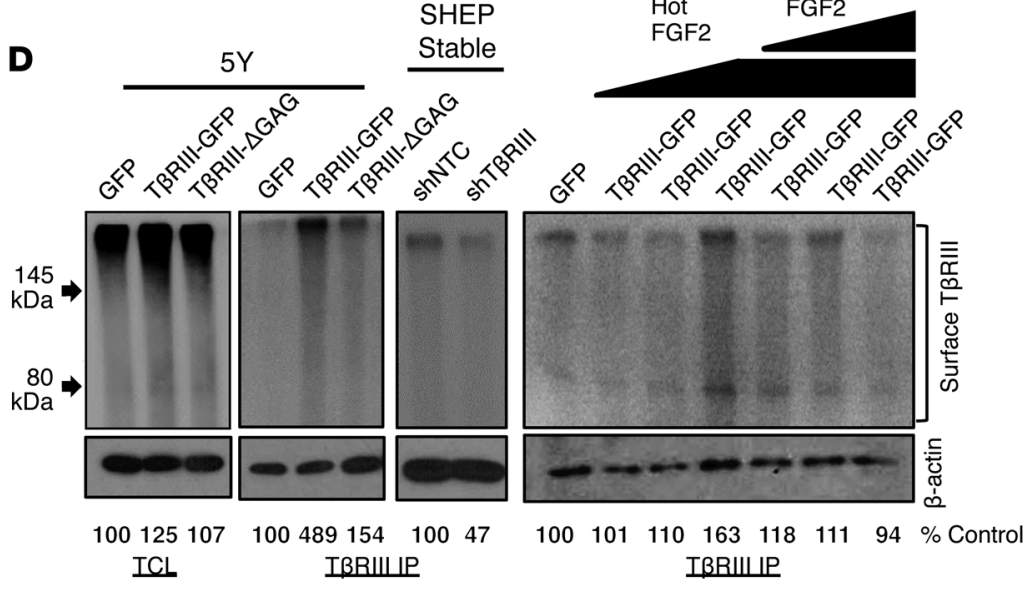

|125 FGF2 Binding

\section{Figure 4}

T $\beta$ RIII promotes neuronal differentiation via FGF2 signaling. (A) Western blots for differentiation markers and graph of neurite analysis using NeuronJ (mean \pm SEM) in 5Y cells expressing nontargeted shRNA or shRNA against T $\beta R I I I$ for 96 hours, with or without $10 \mathrm{ng} / \mathrm{ml}$ FGF2 treatment (gray bars). Densitometry for NF160 normalized to $\beta$-actin is shown as percent control. $P<0.001$ for main effect receptor (2-way ANOVA); $P<0.01$ for main effect FGF2 (2-way ANOVA); interaction $P<0.05$. (B) Western blot for differentiation markers in $5 Y$ cells following 96 hours of adenoviral transduction with GFP control, T $\beta$ RIII-GFP, T $\beta$ RIII-HA, or mutant T $\beta$ RIII-HA lacking GAG chain attachment sites (T $\beta$ RIII- $\triangle$ GAG). Differentiation markers in SHEP cells following 72-hour T $\beta$ RIII knockdown and rescue. Densitometry for NF160 normalized to $\beta$-actin is shown as percent control. NTC, nontargeted control. (C) Western blots in 5Y cells for differentiation markers and phosphorylated and total Erk following 96 hours of transduction and treatment with $1 \mathrm{ng} / \mathrm{ml} \mathrm{FGF2} \mathrm{(gray} \mathrm{bars).} \mathrm{Quantification} \mathrm{of} 3$ independent experiments (mean \pm SEM). $P<0.0001$ for main effect receptor (2-way ANOVA); $P<0.001$ for main effect FGF2 (2-way ANOVA); interaction $P<0.05$. (D) I125 FGF2 binding and crosslinking with total cell lysate (TCL) and T $\beta$ RIII pull-down (T $\beta$ RIII IP) in 5Y and SHEP cell lines. Arrows for total cell lysate mark FGFR1 (145 kDa) and T $\beta$ RIII ( $80 \mathrm{kDa})$. Dose course of I125 FGF2 $(1 \mathrm{ng} / \mathrm{ml}, 5 \mathrm{ng} / \mathrm{ml}, 10 \mathrm{ng} / \mathrm{ml})$; dose course of cold FGF2 (50 ng/ml, $100 \mathrm{ng} / \mathrm{ml}, 500 \mathrm{ng} / \mathrm{ml}) ; \mathrm{GFP}$ condition treated with $10 \mathrm{ng} / \mathrm{ml}$ I $125 \mathrm{FGF} 2$. Densitometry for T $\beta$ RIII normalized to $\beta$-actin is shown as percent control. (E) Coimmunoprecipitation of T $\beta R I I I-H A$ and FGFR1-FLAG in SHEP cells. PAS beads were used as control.

$T \beta R I I I$ promotes differentiation to suppress NB proliferation. To determine the long-term effects of altering T $\beta$ RIII expression in NB cells, we used lentivirus to stably express or knockdown T $\beta$ RIII (Supplemental Figure 2B). Consistent with our previous findings, stably increasing T $\beta$ RIII expression promoted neuronal differentiation, while stable T $\beta$ RIII knockdown decreased differentiation
(Supplemental Figure 6A). Stable high T $\beta$ RIII expression also enhanced FGF2-induced differentiation in a GAG-dependent manner (Supplemental Figure 6A).

Since neuronal differentiation is associated with cell-cycle arrest and tumor regression, we investigated whether stable changes in T $\beta$ RIII expression affected the proliferation of NB cells. We observed 
A

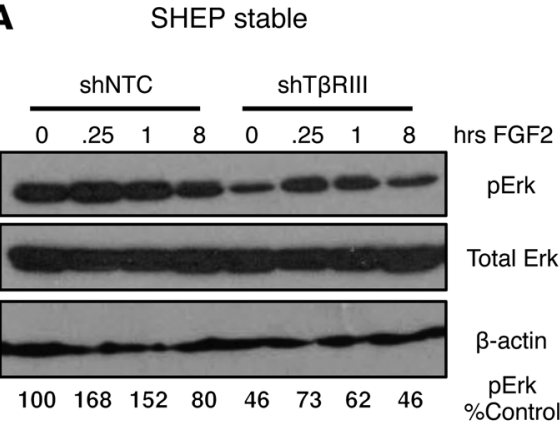

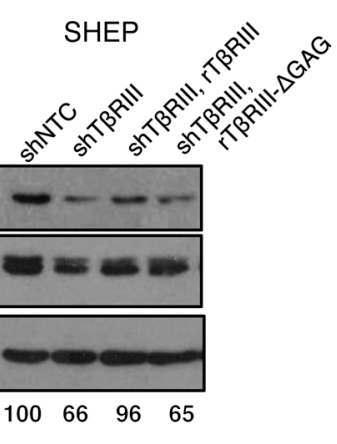

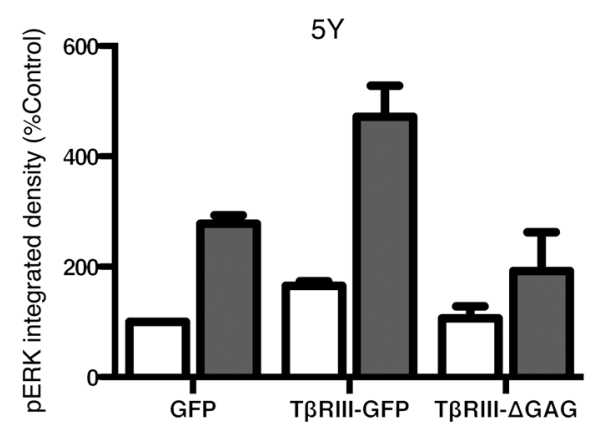

B

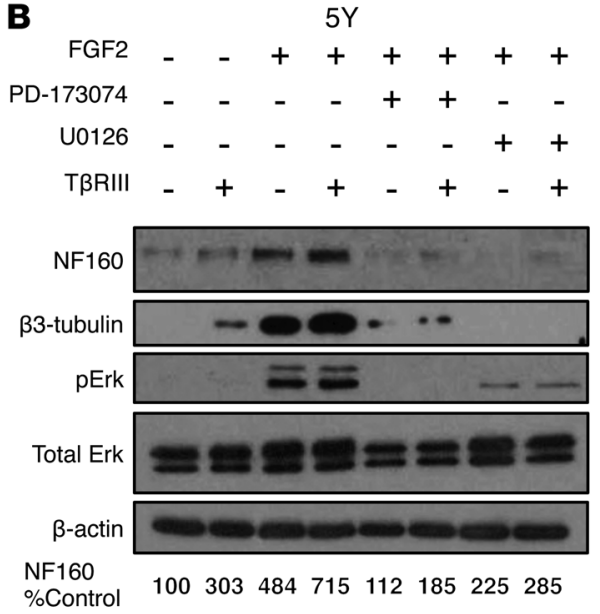

C

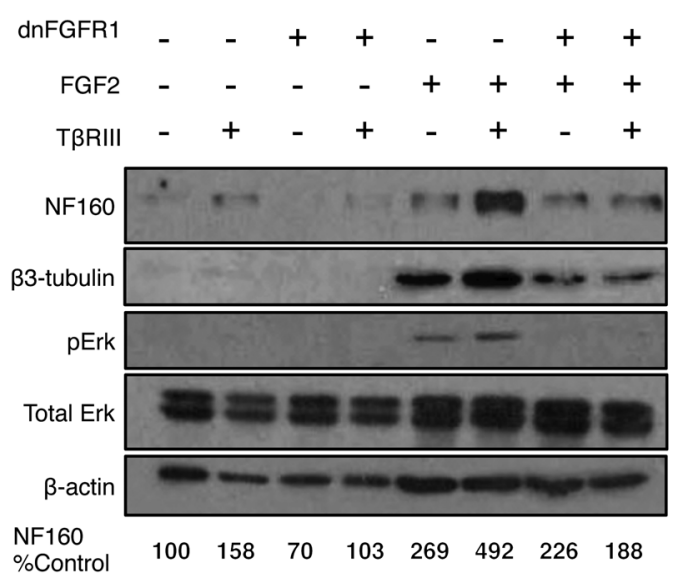

\section{Figure 5}

T $\beta$ RIII enhances FGF2 signaling to promote neuronal differentiation. Cells were treated with doses of $10 \mathrm{ng} / \mathrm{ml}$ FGF2, $1 \mu \mathrm{M} \mathrm{PD}-173074$, and $10 \mu \mathrm{M}$ U0126. (A) Western blot for phosphorylated and total Erk. Differentiation markers after 72-hour T $\beta$ RIII knockdown and rescue with nontargeted shRNA or shRNA against T $\beta$ RIII, with or without $1 \mathrm{ng} / \mathrm{ml} \mathrm{FGF2} \mathrm{treatment} \mathrm{(gray} \mathrm{bars).} \mathrm{Densitometry} \mathrm{for} \mathrm{pErk} \mathrm{normalized} \mathrm{to} \mathrm{total} \mathrm{Erk} \mathrm{is} \mathrm{shown} \mathrm{as}$ percent control. $5 \mathrm{Y}$ cells were transduced for 96 hours. Quantification of densitometry from 4 independent experiments is shown (normalized mean \pm SEM). $P<0.001$ for main effect receptor (2-way ANOVA); $P<0.0001$ for main effect FGF2 (2-way ANOVA); interaction $P<0.05$. (B) Western blots following 96 hours of T $\beta$ RIII transduction and treatment. Densitometry for NF160 normalized to $\beta$-actin is shown as percent control. (C) Western blots following 96 hours of transduction with T $\beta$ RIII or GFP control and dominant-negative FGFR1 (dnFGFR1) or IRES-GFP vector control. GFP fluorescence was used to verify construct expression. Densitometry for NF160 normalized to $\beta$-actin is shown as percent control.

a $35 \%$ decrease in the proliferation index of cells with stable high T $\beta$ RIII expression (Figure 7A and Supplemental Figure 6, B and C). Conversely, stable T $\beta$ RIII knockdown increased proliferation 2 fold (Figure 7A and Supplemental Figure 6B). Microarray and Western blot analysis demonstrated that NB tumors and cell lines with low T $\beta$ RIII expression had increased expression of cell-cycle genes that promote proliferation (Supplemental Figure 1D and Supplemental Figure 6, D and I). Conversely, expression of the cell-cycle regulatory gene $P 21$ was decreased in tumors and cell lines with low T $\beta$ RIII and increased in tumors and cell lines with high T $\beta$ RIII (Figure 7B). Cells with stable high T $\beta$ RIII expression displayed an enhanced p21 response to FGF2 treatment in a GAG-dependent manner, while cells with stable T $\beta$ RIII knockdown exhibited a dramatic attenuation of increased p21 expression following FGF2 treatment (Figure 7B). While p21 expression did not change with NB stage in our meta-analysis of microarray data sets (Supplemental Figure 6E), it correlated with improved prognosis in the Oberthuer data set (ref. 36 and Supplemental Figure 6F). To determine whether T $\beta$ RIII expression affected NB cell proliferation in vivo, we implanted NB cells with stable T $\beta$ RIII knockdown or overexpression (Supplemental Figure 6, G and H) in the mouse adrenal gland
(43). As observed in vitro, T $\beta$ RIII overexpression increased tumor cell differentiation marker expression in a GAG-dependent manner (Figure 7C), whereas tumor cells expressing the T $\beta$ RIII knockdown construct displayed low levels of differentiation markers (Figure 7D). T $\beta$ RIII overexpression dramatically suppressed tumor growth in a GAG-dependent manner (Figure 7C), whereas T $\beta$ RIII knockdown accelerated tumor growth (Figure $7 \mathrm{E}$ ), leading to earlier mortality (Figure 7F). T $\beta$ RIII knockdown also accelerated metastasis to the contralateral adrenal gland and lungs (Figure $7 \mathrm{G}$ and Supplemental Table 2). These results demonstrate that T $\beta$ RIII expression enhances neuronal differentiation to suppress NB cell proliferation, tumor growth, and metastasis.

\section{Discussion}

Here, we present in vitro, in vivo, and clinical data revealing a novel differentiation pathway in NB cells mediated by T $\beta$ RIII coreceptor activity in FGF signaling. Neuronal differentiation represents a validated treatment strategy for NB, yet the growth factor signaling that promotes neuroblast differentiation remains unclear. Dissection of clinically relevant differentiation pathways provides opportunities for therapeutic advances in NB. 
A

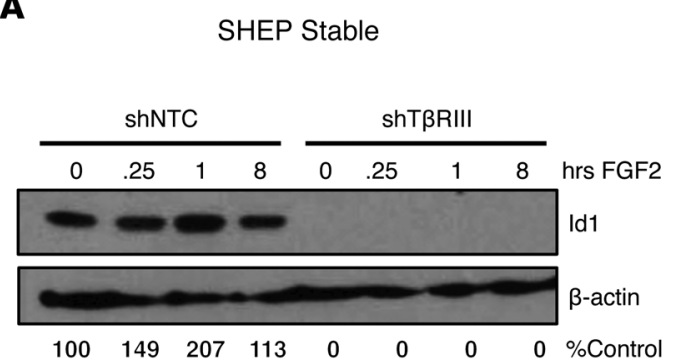

B

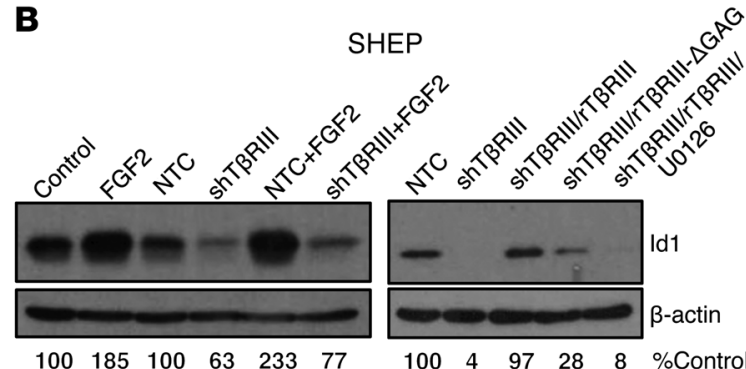

C

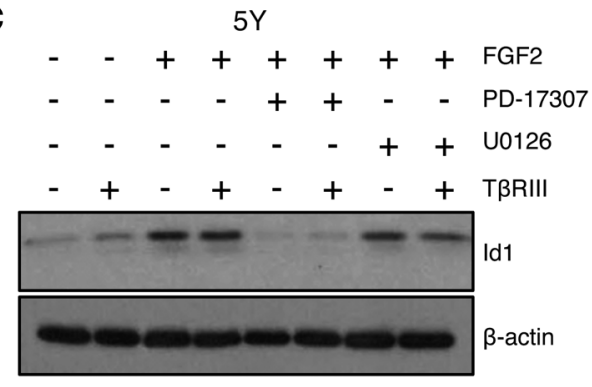

$100194572623 \quad 63 \quad 72 \quad 466 \quad 294$ \%Control
D

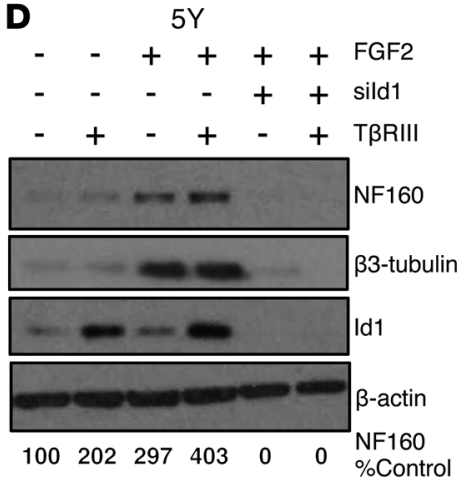

$\mathbf{E}$

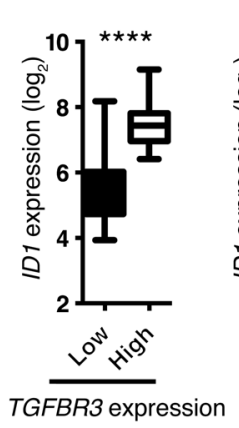

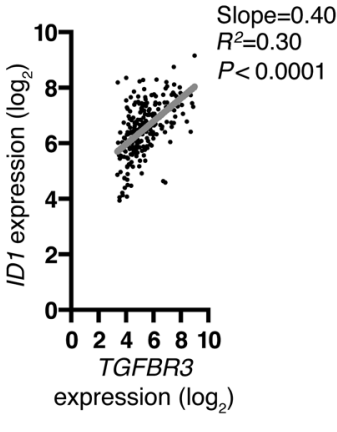

\section{Figure 6}

T $\beta$ RIII and FGF2 cooperate to induce Id1 expression. Cells were treated with doses of $10 \mathrm{ng} / \mathrm{ml} \mathrm{FGF2,} 1 \mu \mathrm{M}$ PD-173074, and 10 $\mu \mathrm{M}$ U0126. (A) Western blot for Id1 in stable SHEP cells serum-starved 24 hours prior to FGF2 treatment. Densitometry analysis for Id 1 normalized to $\beta$-actin is shown as percent control. (B) Western blot for Id1 in SHEP cells transduced and treated with FGF2 for 72 hours. Densitometry for Id1 normalized to $\beta$-actin is shown as percent control. (C) Western blot for Id1 in $5 Y$ cells transduced for 96 hours. Densitometry for Id 1 normalized to $\beta$-actin is shown as percent control. (D) 5 Y cells were transduced for 96 hours with T $\beta$ RIII or GFP control and Id1 siRNA (sild1) or nontargeted control siRNA. Densitometry for NF160 normalized to $\beta$-actin is shown as percent control. (E) Microarray data set expression of ID1 in tumors with low (bottom 10\%) and high (top 10\%) TGFBR3 expression (median [horizontal bars] and interquartile range [boxes]). ${ }^{* \star \star \star} P<0.0001$ (Mann-Whitney). Linear regression analysis of ID1 expression, which was dependent on TGFBR3 expression, in the microarray data set.

Consistent with prior reports $(16,20,21)$, we demonstrate that T $\beta$ RIII expression is decreased at both the message and protein level in NB. As low T $\beta$ RIII expression is associated with poor event-free survival (Figure 1D and Supplemental Figure 1A), $\mathrm{T} \beta \mathrm{RIII}$ could be used as a prognostic biomarker. Moreover, T $\beta$ RIII expression can risk stratify both early-stage and $M Y C N$-amplified NB (Figure 1E and Figure 2A), further supporting its utility as a prognostic biomarker.

As demonstrated here, epigenetic suppression of T $\beta$ RIII is a novel downstream effector for the MYCN oncogene, which is itself a poor therapeutic target. While the precise mechanism of epigenetic suppression remains to be defined, $M Y C N$-mediated T $\beta$ RIII suppression can be reversed by inhibition of HDACs (Figure 2G). Thus, these studies provide a mechanistic rationale for clinical trials already underway using HDAC inhibitors in patients with NB and provide a potential biomarker for response to therapy in these trials.

T $\beta$ RIII-induced neuronal differentiation is dependent upon functional FGFR1, Erk MAPK, and Id1 (Figures 3-6), with FGF2 treatment enhancing this differentiation pathway. While FGF2 and Erk MAPK signaling have been shown to promote tumor cell proliferation and disease progression in a variety of cancers (44, 45 ), our data identify an unexpected role for this ubiquitous signaling pathway in suppressing tumor growth.

Recent evidence suggests that Erk signaling is also critical for retinoic acid- and $\alpha$-lipoic acid-induced NB differentiation and cell-cycle exit via upregulation of p21 $(31,32)$, suggesting activity of this differentiation pathway in response to multiple stimuli. Interestingly, of the signaling components involved in the differentiation pathway we have identified (including FGF2, FGFR1, T $\beta$ RIII, Erk1/2, Id1, and p21), only T $\beta$ RIII expression varies by stage of disease, while correlating with improved prognosis, highlighting its importance (Figure 1, Supplemental Figure 6, E and F; and negative data not shown). Likewise, in vitro overexpression and knockdown studies demonstrate that T $\beta$ RIII expression is critical to neuronal differentiation and $\mathrm{p} 21$ induction in response to FGF2 treatment in NB cells (Figure 4A and Figure 7B). Thus, restoring T $\beta$ RIII, either through HDAC inhibition or potentially through the administration of recombinant soluble T $\beta$ RIII, may be beneficial in the clinical treatment of NB. To this end, we have demonstrated that soluble T $\beta$ RIII can also induce differentiation in NB models (Erik Knelson, unpublished observations). These studies also urge caution in the clinical development of nonspecific tyrosine kinase inhibitors for the treatment of NB. Off-target inhibition of neuroblast differentiation, which has been observed in preclinical models (46), could lead to drug resistance and disease progression.

Contrary to well-established in vitro markers of neuroblast differentiation, identification of in vivo markers has been challenging. Expression of individual genes is often unreliable (47), and some in vitro differentiation markers, such as tyrosine hydroxylase 

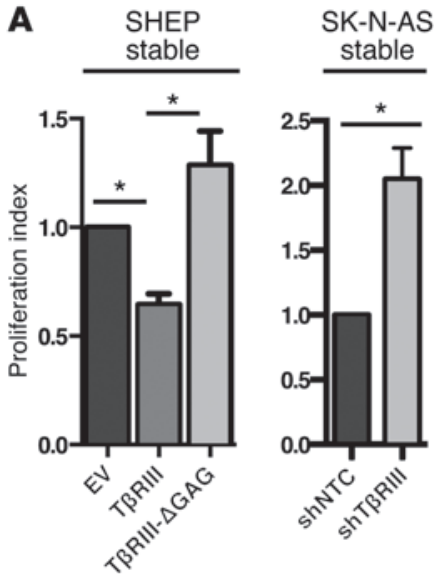

B

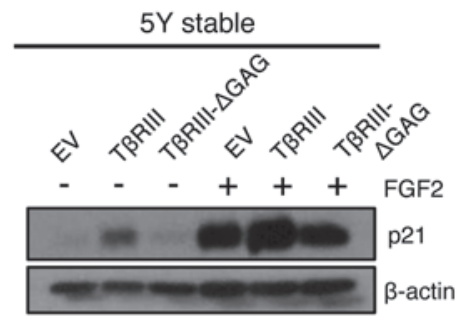

$100201100397582385 \%$ Control

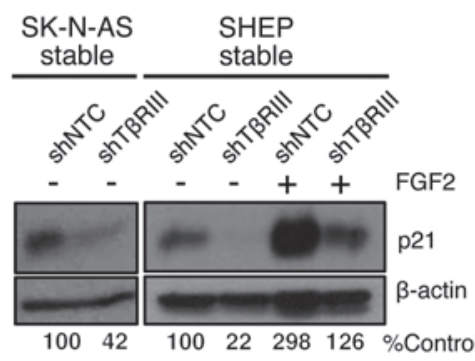

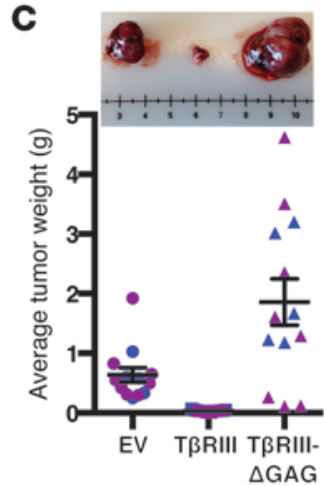
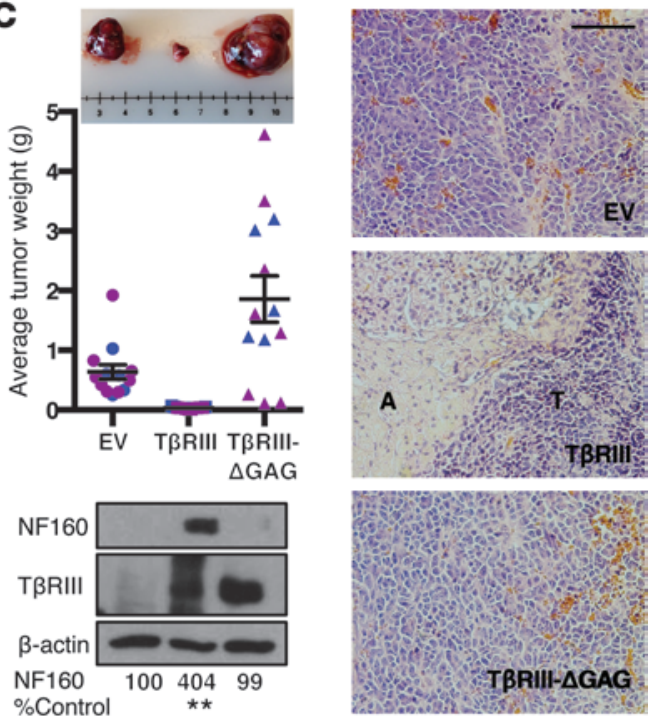

D

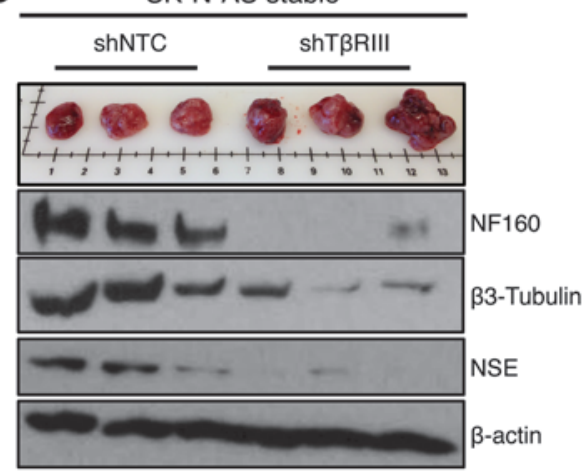

E

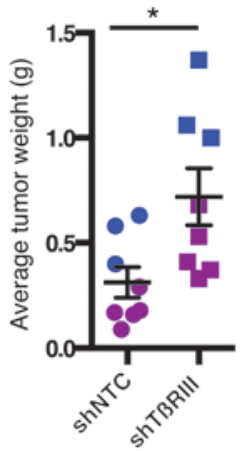

$\mathbf{F}$

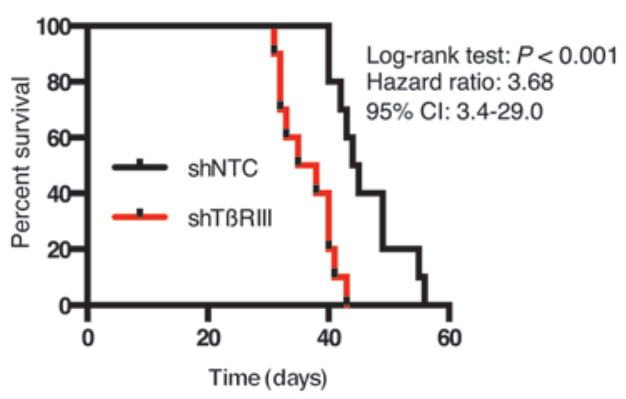

G

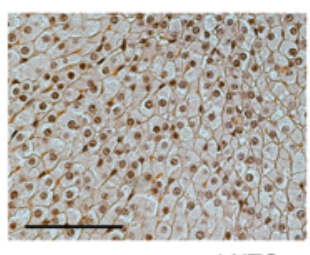

ShNTC

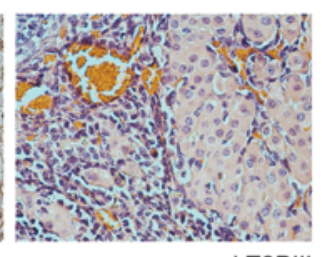

$\operatorname{shT} \beta R I I I$

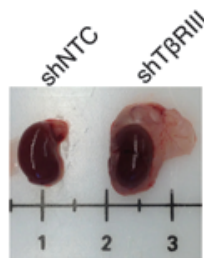

Figure 7

T $\beta$ RIII promotes differentiation to suppress NB proliferation. $5 Y$, SHEP, and SK-N-AS cells selected for stable expression of T $\beta R$ III, T $\beta R$ III- $\Delta$ GAG, empty vector control (EV), shRNA to T $\beta$ RIII (shT $\beta$ RIII), or nontargeted shRNA control (shNTC). (A) Proliferation index from 3 replicates (mean \pm SEM) of thymidine incorporation, normalized to empty vector or nontargeted shRNA control lines. $P<0.01$ (ANOVA); ${ }^{*}<0.05$ (1-sample $t$ test and 2-tailed Student's $t$ test). (B) Western blot for p21 in stable cell lines, with or without FGF2 treatment (1 ng/ml for $5 \mathrm{Y}, 10 \mathrm{ng} / \mathrm{ml}$ for SHEP). Densitometry for p21 normalized to $\beta$-actin is shown as percent control. (C) 5 Y stable orthotopic xenografts (13 mice per group). Tumor weights (mean \pm SEM) and images (scale bar in $\mathrm{cm}$ ) after 7 weeks of growth. Different symbol colors represent different cohorts. $P<0.0001$ (1-way ANOVA); pairwise comparisons $P<0.0001 \mathrm{EV}$ vs. T $\beta$ RIII, $P<0.05 \mathrm{EV}$ vs. T $\beta$ RIII- $\triangle$ GAG (Mann-Whitney) Western blots of tumor lysates. Average NF160 densitometry from 3 replicates normalized to $\beta$-actin is shown as percent control. ${ }^{\star \star} P<0.01$ (1-sample $t$ test). H\&E staining of tumors from each group. T, tumor; A, host adrenal cells. Scale bar: $50 \mu \mathrm{M}$. (D) SK-N-AS stable orthotopic xenografts. Tumor images after 4 weeks of growth (scale bar in $\mathrm{cm}$ ). Western blot of tumor lysates for differentiation markers. (E) Tumor weights at 4 weeks (mean \pm SEM). Different symbol colors represent different cohorts. ${ }^{*} P<0.05$ (Mann-Whitney). (F) Kaplan-Meier survival analysis (10 mice per group). (G) H\&E-stained contralateral adrenal glands from mice at 4 weeks (scale bar: $50 \mu M$ ). Photograph of macroscopic metastasis to the contralateral adrenal gland at the 4-week end point (scale bar in $\mathrm{cm}$ ).

and neuron-specific enolase, are markers of metastasis (48) and poor prognosis $(49,50)$ when used clinically. In our meta-analysis of a microarray data set, none of the in vitro differentiation markers we used elsewhere correlated with stage of disease or TGFBR3 expression (data not shown). However, elevated levels of the transcription master regulator SOX10 in NB tumors correlated with high T $\beta$ RIII expression (Figure $3 \mathrm{H}$ ). As SOX10 expression is critical to neuronal differentiation in development (40) and is associ- 
ated with more differentiated NB (41), the investigation of SOX10 and TGFBR3 expression may provide useful biomarkers for differentiation therapy response. In addition, we show that TGFBR3 expression tracks with a 9-gene signature of differentiated NB cells previously shown to predict response to differentiating agents (Supplemental Figure 1C and ref. 47).

Based on Scatchard analysis of binding, the T $\beta$ RIII binding affinity for FGF2 $\left(K_{\mathrm{d}} \sim 100 \mathrm{pM}\right)$ is on the same order of magnitude for binding of FGF2 to FGF receptors (51), suggesting high-affinity FGF2 binding to T $\beta$ RIII (Supplemental Figure 4C). While the ability of T $\beta$ RIII to bind FGF2 via GAG chains has been previously reported (33) and T $\beta$ RIII has been shown to promote FGF2-mediated biology in epicardial cells (34), this is the first demonstration of T $\beta$ RIII effects on FGF signaling and biology in NB. Additionally, we demonstrate for the first time an interaction between T $\beta$ RIII and FGFR1, which can induce Erk MAPK signaling and promote differentiation in the absence of ligand (Figure 4E, Supplemental Figure 3B, Supplemental Figure 4E, and Supplemental Figure 5, C and D). Given the ubiquity of T $\beta$ RIII expression and FGF signaling, it is likely this coreceptor activity occurs in other contexts where T $\beta$ RIII and FGF2 have demonstrated roles. Based on the mechanism of signaling crosstalk via GAG chains, it is also possible that other proteoglycan coreceptors, including the glypicans and syndecans, could have similar activity to that of T $\beta$ RIII in NB. The role of other proteoglycan coreceptors in NB is currently being explored.

In conclusion, we demonstrate a novel and clinically relevant mechanism for neuroblast differentiation. Further, these studies identify T $\beta$ RIII expression as a prognostic biomarker for patients with early-stage and $M Y C N$-amplified NB, while providing mechanistic support for the use of HDAC inhibitors and recombinant soluble T $\beta$ RIII in clinical trials. More generally, our work provides preclinical rationale for targeting differentiating growth factors and receptors in the treatment of NB.

\section{Methods}

Microarray data set analysis. To generate our microarray data set, we downloaded 5 publicly available NB data sets from GEO (GSE12460, GSE16237, GSE13141, GSE21713, and GSE27608), which include data generated on multiple Affymetrix platforms. Microarray data were RMA preprocessed $(52,53)$, and all data were $\log _{2}$ transformed. Human Exon 1.0 ST array gene level probes were matched to their best-match HG-U133 Plus 2.0 probe set as described previously (54). To minimize batch effects from each of the 5 separate data sets, we used ComBat software as described previously (55). We then queried our data set using the gene probes listed in Supplemental Table 1. Survival analysis was conducted using the oncogenomics website (http://home.ccr.cancer.gov/oncology/oncogenomics/), specifically the Oberthuer (36) and NB prognosis (37) data sets.

T $\beta$ RII immunobistochemistry. NB tissue samples were obtained from the Children's Oncology Group (COG) Biorepository with approval from the COG Neuroblastoma Biology Subcommittee. T $\beta$ RIII immunohistochemistry was conducted using a biotin-free protocol from BioCare Medical according to the manufacturer's instructions. Briefly, patient sample slides were deparaffinized, rehydrated, and blocked with Peroxidazed 1 (PX968G, Biocare Medical) and Background Punisher (BP974G, Biocare Medical), before incubation with a custom-made rabbit antibody to the cytoplasmic domain of T $\beta$ RIII, as described previously $(56,57)$. This was followed by sequential treatment with an alkaline phosphatase polymer system and the Warp Red chromogen (M3R533G and WR806H, respectively; Biocare Medical). Semiquantitative analysis was performed independently by 2 blinded investigators using a 3-tiered scoring system ( 0 , no staining; 1 , staining present but minimal; 2 , moderate to dark staining). Discrepancies between the 2 investigators were discussed and reconciled (<10 samples).

Cell culture and reagents. SK-N-SH-SY5Y (5Y; CRL-2266) and SK-N-BE (2) (BE2; CRL-2271) cells were purchased from ATCC and grown in a 1:1 mixture of Eagle minimum essential medium and Ham's F12 with 10\% fetal bovine serum. SK-N-SH-SHEP (SHEP; gift of M.A. Armstrong, Duke University, Durham, North Carolina, USA), SK-N-AS (ATCC CRL-2137), SK-NSH (ATCC HTB-11), S16 (ATCC CRL-2941), and SK-N-AS-MYCNER and SHEP-21N (gifts of Linda Valentijn, University of Amsterdam, Amsterdam, The Netherlands; ref. 39) cells were grown in Dulbecco modified Eagle minimum essential medium with $10 \%$ fetal bovine serum. All cells were grown at $37^{\circ} \mathrm{C}$ in $5 \% \mathrm{CO}_{2}$. Human basic fibroblast growth factor (no. 8910) and the MEK 1/2 inhibitor U0126 (no. 9903) were purchased from Cell Signaling. The MEK 1/2 inhibitor CI-1040 (S1020) was purchased from Selleck Chemicals. The FGFR inhibitor SU5402 (sc-204308) was purchased from Santa Cruz Biotechnology Inc. The FGFR inhibitor PD-173074 (P2499), the p38 inhibitor SB203580 (S8307), and the Alk 4/5/7 inhibitor SB431542 (S4317) were purchased from Sigma-Aldrich. The neutralizing TGF- $\beta 1$ antibody 1D11 (MAB 1835) was purchased from R\&D Systems. The neutralizing FGF2 antibody (catalog no. 05-117) was purchased from Millipore and used at a concentration of $5 \mu \mathrm{g} / \mathrm{ml}$ per manufacturer's instructions. The BMP inhibitor dorsomorphin (catalog no. 3093) was purchased from Tocris. The Alk 2/3 inhibitor LDN193189 was a gift from Paul Yu (Massachusetts General Hospital, Boston, Massachusetts, USA; ref. 58).

DNA constructs. All T $\beta$ RIII and T $\beta$ RIII shRNA constructs used in this study have been described previously $(57,59-63)$. T $\beta$ RIII-HA consists of the fulllength human T $\beta$ RIII sequence with the HA sequence at the $\mathrm{N}$ terminus, within the pcDNA 3.1 vector (62). T $\beta$ RIII-GFP consists of the full-length human T $\beta$ RIII sequence inserted in the bicistronic pEGFP vector (61). rT $\beta$ RIII consists of the rat T $\beta$ RIII sequence with HA tag in the pcDNA 3.1 vector (57). T $\beta$ RIII- $\triangle$ GAG consists of T $\beta$ RIII-HA, with serine-to-alanine point mutations at amino acids 534 and 545 to prevent GAG attachment $(33,59,61,62)$. T $\beta$ RIII-cyto consists of T $\beta$ RIII-HA with a truncation of the cytoplasmic domain $(59,63)$. Adenoviral constructs were used at a MOI of 10 particles per cell. T $\beta$ RIII adenoviral shRNA constructs were used at an MOI of 50 particles per cell. Lentiviral vectors consisted of the same construct as used in adenoviral vectors cloned into a pSMPUW-Neo backbone (T $\beta$ RIII constructs) or a pLKO.1-puro backbone (T $\beta$ RIII shRNA construct and nontargeted control). Transient DNA transfections were performed using lipofectamine (Invitrogen) according to the manufacturer's instructions.

Id1 siRNA (sc29356) and control siRNA (sc37007) were purchased from Santa Cruz Biotechnology Inc. and used according to the manufacturer's instructions. pWZL Neo Myr Flag FGFR1 (Addgene plasmid no. 20486) was a gift of Jean Zhao and William Hahn (Dana-Farber Cancer Institute, Boston, Massachusetts, USA) (64). The dnFGFR1 plasmid with a GFP reporter (pCCALL2 dominant-negative FGFRI IRES EGFP) was a gift of Margaret Kirby and Harriett Stadt (Duke University) (42).

Neurite analysis. Neurites were measured from phase-contrast images taken with a Nikon inverted microscope at $\times 10$ magnification using the NIH ImageJ plug-in NeuronJ (65). Three images were taken of each condition at each time point, and all visible neurites (thin shafts extending outward from the cell body) were measured (70-150 neurites per field).

Immunoprecipitation, Western blotting, and flow cytometry. Immunoprecipitation and Western blotting were performed using standard techniques as described previously $(66,67)$. Each experiment was conducted at least 3 separate times. Antibodies for differentiation and signaling markers were purchased from Cell Signaling: neurofilament 160 kDa (NF160) (no. 2838), $\beta 3$-tubulin (no. 5568), tyrosine hydroxylase (no. 2792), neuron-specific enolase (no. 9536), GAP43 (no. 5307), phospho-Erk 1/2 (pErk) T202/ 
Y204 (no. 9101), Erk 1/2 (no. 4695), p21 (no. 2946), MYCN (no. 9405), acetyl lysine (no. 9441), and cyclin D1 (no. 2926). Id1 antibody (sc488) was purchased from Santa Cruz Biotechnology Inc.

The lysis buffer for coimmunoprecipitation experiments contained $0.75 \%$ NP40 and 2 nM EDTA ( $0.1 \%$ NP40 for endogenous protein experiments). The HA antibody (HA.11 clone 16B12 MMS-101P) was purchased from Covance, and the FLAG antibody (F3165, clone M2) was purchased from Sigma-Aldrich. Both antibodies were used at a concentration of 10 $\mu \mathrm{g} / \mathrm{ml}$ for immunoprecipitation, as per manufacturer's instructions. For endogenous immunoprecipitation, T $\beta$ RIII antibody (AF-242-PB, R\&D Systems) and FGFR1 antibody (9740, Cell Signaling) were used. Lysates were precleared in PAS beads (PGS for the goat TRRIII antibody) for 2 hours and incubated overnight with beads and pull-down antibody.

T $\beta$ RIII flow cytometry was conducted using the R\&D Systems antibody following the manufacturer's instructions and using a 488-GFP fluorophore-tagged anti-goat secondary antibody and Accuri C6 flow cytometer.

Iodinated ligand binding and crosslinking. Iodinated TGF- $\beta 1$ binding and crosslinking was conducted with T $\beta$ RIII pull down using a goat antibody to the extracellular domain (AF-242-PB, R\&D Systems) in order to identify functional surface receptor expression as described previously $(56,59)$. Iodinated FGF2 binding and crosslinking were conducted as with TGF- $\beta 1$, with the following changes: $0.5 \%$ NP40 lysis buffer was used instead of RIPA and 30 minutes of crosslinking with $0.02 \%$ DSS was used instead of 15 minutes with $0.1 \%$ DSS. Both iodinated TGF- $\beta 1$ (NEX2670) and iodinated FGF2 (NEX268) were purchased from Perkin Elmer.

ChIP. ChIP analysis was performed using the ChIP-IT Express Chromatin Immunoprecipitation Kit (Active Motif) according to the manufacturer's instructions. Briefly, chromatin was sheared ( $\sim 500$ bp average length) by sonication with a Branson Sonifier 250 (output control 1.5; duty cycle 25\%; 10 cycles of 20 -second pulses at 30 -second intervals). Sheared cross-linked chromatin was rotated at $4{ }^{\circ} \mathrm{C}$ overnight with protein $\mathrm{G}$ magnetic beads and MYCN (OP13, Calbiochem) or mouse IgG (015-000-003, Jackson ImmunoResearch Laboratories Inc.). Following chromatin elution, cross-link reversal, and proteinase $\mathrm{K}$ digestion, samples were purified using the QIAquick PCR Purification Kit (28104, Qiagen). PCR products were analyzed by quantitative RT-PCR using iQ SYBR Green Supermix (170-8882, Bio-Rad) and normalized to input controls. The following primers were used in the ChIP assays: human T $\beta$ RIII Sp-1 +1 kB, 5'-GCAGCAAGTTGGAGGAAAGC-3' and 5'-GTCCGGATGGCGTAGTTTTG-3' (102 bp); human T $\beta$ RIII Sp-1 $+3 \mathrm{kB}, 5^{\prime}$-TCCTTTAACTGACACAATGGCATG-3' and $5^{\prime}$-AGGAAACAGCTTGGGGTTGG-3' (103 bp); human T $\beta$ RIII Sp-1 +5 kB, 5'-TACATAATATGGGGCCGGGC-3' and 5'-GTAGAGACGGGGCTTCACTG-3' (129 bp); human T $\beta$ RIII Sp-1 +7 kB, 5'-TCAACATAAAAGAACCACCACCA-3' and $5^{\prime}$-ACAAGAGCCCCAGAACCATG-3' (127 bp); human T $\beta$ RIII Sp-1 -2 $\mathrm{kB}, 5^{\prime}$-CTGACAAATGCCACCACGC-3' and 5'-AGGCAGGCGAATCTCTTGAG-3' (125 kB); and human T $\beta$ RIII Sp-1 -20 kB, 5'-AGATAATTCTGGACGGGCGC-3' and 5'-TGTTGGCCAGAACAGTCTCG-3' (107 bp). As a negative control, human T $\beta$ RIII primers were designed $90 \mathrm{kB}$ downstream of the transcriptional start site, $5^{\prime}$-TGTCCTGAATCTCCGCACTG-3' and 5'-GTGGTGGATGTGGACTGAGG-3' (97 bp). As a positive control, primers toward the Bmi1 promoter were used as previously described (68).

Proliferation assays. Tritiated thymidine incorporation was used to assess cell proliferation as described previously (67). Proliferation indices (normalized to control $=1.0$ ) were calculated and averaged for each of 3 individual experiments at different cell densities in order to examine proliferation differences across a range of cellular confluence. Cells were plated in a 96-well plate at a concentration of 400 to 5,000 cells per well (SHEP cells) or 5,000 to 10,000 cells per well (SK-N-AS cells). Each condition was plated in triplicate overnight prior to a 4-hour [3H]thymidine pulse $(1 \mu \mathrm{Ci}$; Amersham Biosciences/GE Healthcare). Cells were washed with PBS and
$5 \%$ trichloroacetic acid prior to lysis with $0.1 \mathrm{~N} \mathrm{NaOH}$. Incorporation of $[3 \mathrm{H}]$ thymidine was determined by scintillation counting.

Orthotopic xenograft. Antibiotic-selected stable cell lines were implanted orthotopically ( 2 million cells per mouse in $20 \mu \mathrm{l}$ DMEM) in the left adrenal capsule of 8-week-old female beige/SCID mice (Charles River Laboratories) as described previously (43). Mice were housed under pathogen-free conditions on a 12-hour-light/dark cycle. Animals were monitored closely for tumor growth and signs of illness and sacrificed at humane end points. For the surgical procedure, anesthetized mice underwent left subcostal laparotomy. Gentle retraction of the spleen exposed the adrenal gland for injection using a 23-gauge needle (7804-07, Hamilton Company; 2-inch PT2) on a $25-\mu 1$ syringe (no. 702, Hamilton Company). Peritoneal and cutaneous incisions were closed in 2 layers with 4.0 silk suture (Sharpoint $18 \mathrm{~mm} \mathrm{DA-}$ $2187 \mathrm{~N}$; Surgical Specialties Corp.).

Statistics. All clinical and xenograft data were analyzed using nonparametric statistics (Kruskal-Wallis global test with Mann-Whitney post-hoc tests) and presented as median, upper, and lower quartile. Survival curves were analyzed with log-rank statistics. In vitro experiments were analyzed using parametric statistics (ANOVA global test with Bonferroni-corrected 2-tailed Student's $t$ tests as post-hoc tests) and presented as mean \pm SEM. In cases in which data were normalized to control, 1-sample Student's $t$ test was used with an expected value of 1 or $100 \%$ in order to decrease the likelihood of a type I error. To examine the statistical interaction between receptor expression and ligand treatment, 2-way ANOVA was performed with specific interest in the interaction term. The isolated effect of each individual variable (represented by an ANOVA $P$ value) was also noted in the figures and referred to as main effect receptor or main effect FGF2. For all experiments, significance was set at $P<0.05$. Linear regression was performed on selected microarray data, with the slope and $P$ value for the line of best fit reported as well as the $r^{2}$ value for the relationship. All statistical analyses were conducted with GraphPad Prism version 6.00 (GraphPad Software).

Study approval. All patient samples were deidentified, and the project was exempted by the Duke University Health System Institutional Review Board (protocol ID 00034541). All animal procedures were approved by the Duke University Institutional Animal Care and Use Committee (protocol A278-11-11).

\section{Acknowledgments}

We thank Michael Hogarty, the Children's Oncology Group Neuroblastoma Biology Subcommittee, Wendy London, and Evan Plunkett for providing patient tissue and serum samples. We thank Linda Valentijn, Paul Yu, Harriett Stadt, Mary Hutson, Margaret Kirby, and Lisa Crose for providing reagents. We thank Lindsey Morgan and Terri Lucas for coordinating our animal facility use. We thank Julie Fuller for tissue processing. We are grateful to Tam How, Catherine Gatza, Alison Meyer, Alisha Holtzhausen, Catherine Lavau, Rebekah Moehring, Jennifer Elderbroom, Rachel Hesler, and Jasmine Nee for technical assistance and Cheryl Alles for superior clerical assistance. We are grateful to Daniel Wechsler, Dona Chikaraishi, Christopher Kontos, and Julio Ramirez for invaluable mentoring throughout this project. This work was supported in part by NIH grants F30 CA168043-01 (to E.H. Knelson), R01-CA136786 (to G.C. Blobe), and R01-CA135006 (to G.C. Blobe).

Received for publication March 1, 2013, and accepted in revised form August 8, 2013.

Address correspondence to: Gerard C. Blobe, Duke University Medical Center, Box 91004, Durham, North Carolina 27708, USA. Phone: 919.668.1359; Fax: 919.681.6906; E-mail: gerard.blobe@duke.edu. 
1. National Cancer Institute. Surveillance, Epidemiology and End Results (SEER) Database. NIH Web site. http://seer.cancer.gov/. Accessed August 30, 2013.

2. Mullassery D, Dominici C, Jesudason EC, McDowell HP, Losty PD. Neuroblastoma: contemporary management. Arch Dis Child Educ Pract Ed. 2009;94(6):177-185.

3. Maris JM, Hogarty MD, Bagatell R, Cohn SL. Neuroblastoma. Lancet. 2007;369(9579):2106-2120.

4. De Bernardi B, et al. Retrospective study of childhood ganglioneuroma. J Clin Oncol. 2008; 26(10):1710-1716.

5. Retrosi G, et al. Morbidity after ganglioneuroma excision: is surgery necessary? Eur J Pediatr Surg. 2011;21(1):33-37.

6. Janoueix-Lerosey I, Schleiermacher G, Delattre O. Molecular pathogenesis of peripheral neuroblastic tumors. Oncogene. 2010;29(11):1566-1579.

7. Maris JM. Recent advances in neuroblastoma. NEngl J Med. 2010;362(23):2202-2211.

8. Brodeur GM. Neuroblastoma: biological insights into a clinical enigma. Nat Rev Cancer. 2003; 3(3):203-216.

9. Seeger RC, et al. Association of multiple copies of the $\mathrm{N}$-myc oncogene with rapid progression of neuroblastomas. N Engl J Med. 1985; 313(18):1111-1116.

10. Schwab M, et al. Amplified DNA with limited homology to myc cellular oncogene is shared by human neuroblastoma cell lines and a neuroblastoma tumour. Nature. 1983;305(5931):245-248.

11. Westermark UK, Wilhelm M, Frenzel A, Henriksson MA. The MYCN oncogene and differentiation in neuroblastoma. Semin Cancer Biol. 2011;21(4):256-266.

12. Bell E, Chen L, Liu T, Marshall GM, Lunec J, Tweddle DA. MYCN oncoprotein targets and their therapeutic potential. Cancer Lett. 2010;293(2):144-157.

13. Matthay KK, et al. Long-term results for children with high-risk neuroblastoma treated on a randomized trial of myeloablative therapy followed by 13-cis-retinoic acid: a children's oncology group study. J Clin Oncol. 2009;27(7):1007-1013.

14. Edsjo A, Holmquist L, Pahlman S. Neuroblastoma as an experimental model for neuronal differentiation and hypoxia-induced tumor cell dedifferentiation. Semin Cancer Biol. 2007;17(3):248-256.

15. Gilbert SF. The neural crest. In: Developmental Biology. Sunderland, Massachusetts, USA: Sinauer Associates; 2000.

16. Iolascon A, et al. Reduced expression of transforming growth factor-beta receptor type III in high stage neuroblastomas. Br J Cancer. 2000; 82(6):1171-1176.

17. McCune BK, Patterson K, Chandra RS, Kapur S, Sporn MB, Tsokos M. Expression of transforming growth factor-beta isoforms in small round cell tumors of childhood. An immunohistochemical study. Am J Pathol. 1993;142(1):49-58.

18. Mestdagh P, et al. The miR-17-92 microRNA cluster regulates multiple components of the TGF-beta pathway in neuroblastoma. Mol Cell. 2010;40(5):762-773.

19. Turco A, et al. Increased TGFbeta type II receptor expression suppresses the malignant phenotype and induces differentiation of human neuroblastoma cells. Exp Cell Res. 2000;255(1):77-85.

20. Takita J, et al. Gene expression profiling and identification of novel prognostic marker genes in neuroblastoma. Genes Chromosomes Cancer. 2004;40(2):120-132.

21. De Preter K, et al. Human fetal neuroblast and neuroblastoma transcriptome analysis confirms neuroblast origin and highlights neuroblastoma candidate genes. Genome Biol. 2006;7(9):R84.

22. Rogers SL, Cutts JL, Gegick PJ, McGuire PG, Rosenberger C, Krisinski S. Transforming growth factor-beta 1 differentially regulates proliferation, morphology, and extracellular matrix expression by three neural crest-derived neuroblastoma cell lines. Exp Cell Res. 1994;211(2):252-262.

23. Scarpa S, et al. Transforming growth factor beta regulates differentiation and proliferation of human neuroblastoma. Exp Cell Res. 1996; 229(1):147-154.

24. Gomez-Santos C, Ambrosio S, Ventura F, Ferrer I, Reiriz J. TGF-beta1 increases tyrosine hydroxylase expression by a mechanism blocked by BMP-2 in human neuroblastoma SH-SY5Y cells. Brain Res. 2002;958(1):152-160.

25. Du Y, Yip H. Effects of bone morphogenetic protein 2 on Id expression and neuroblastoma cell differentiation. Differentiation. 2010;79(2):84-92.

26. Lavenius E, Parrow V, Nanberg E, Pahlman S. Basic FGF and IGF-I promote differentiation of human SH-SY5Y neuroblastoma cells in culture. Growth Factors. 1994;10(1):29-39.

27. Guillemot F, Zimmer C. From cradle to grave: the multiple roles of fibroblast growth factors in neural development. Neuron. 2011;71(4):574-588.

28. Kim MS, et al. Fibroblast growth factor 2 induces differentiation and apoptosis of Askin tumour cells. J Pathol. 2004;202(1):103-112.

29. Higgins $S$, et al. Fibroblast growth factor 2 reactivates $\mathrm{G} 1$ checkpoint in SK-N-MC cells via regulation of $\mathrm{p} 21$, inhibitor of differentiation genes (Id1-3), and epithelium-mesenchyme transition-like events. Endocrinology. 2009;150(9):4044-4055.

30. Passiatore G, Gentilella A, Rom S, Pacifici M, Bergonzini V, Peruzzi F. Induction of Id-1 by FGF-2 involves activity of EGR-1 and sensitizes neuroblastoma cells to cell death. J Cell Physiol. 2011; 226(7):1763-1770.

31. Wang X, et al. Essential role of ERK activation in neurite outgrowth induced by alpha-lipoic acid. Biochim Biophys Acta. 2011;1813(5):827-838.

32. Qiao J, et al. PI3K/AKT and ERK regulate retinoic acid-induced neuroblastoma cellular differentiation. Biochem Biophys Res Commun. 2012;424(3):421-426.

33. Andres JL, DeFalcis D, Noda M, Massague J. Binding of two growth factor families to separate domains of the proteoglycan betaglycan. J Biol Chem. 1992;267(9):5927-5930.

34. Sanchez NS, et al. The cytoplasmic domain of TGFbetaR3 through its interaction with the scaffolding protein, GIPC, directs epicardial cell behavior. Dev Biol. 2011;358(2):331-343

35. Gatza CE, Oh SY, Blobe GC. Roles for the type III TGF-beta receptor in human cancer. Cell Signal. 2010;22(8):1163-1174.

36. Oberthuer A, et al. Customized oligonucleotide microarray gene expression-based classification of neuroblastoma patients outperforms current clinical risk stratification. J Clin Oncol. 2006;24(31):5070-5078.

37. Wei JS, et al. Prediction of clinical outcome using gene expression profiling and artificial neural networks for patients with neuroblastoma. Cancer Res. 2004;64(19):6883-6891.

38. Brodeur GM, Seeger RC, Schwab M, Varmus HE, Bishop JM. Amplification of $\mathrm{N}$-myc in untreated human neuroblastomas correlates with advanced disease stage. Science. 1984;224(4653):1121-1124.

39. Valentijn LJ, Koppen A, van Asperen R, Root HA, Haneveld F, Versteeg R. Inhibition of a new differentiation pathway in neuroblastoma by copy number defects of $\mathrm{N}-\mathrm{myc}, \mathrm{Cdc} 42$, and $\mathrm{nm} 23$ genes. Cancer Res. 2005;65(8):3136-3145.

40. Kelsh RN. Sorting out Sox10 functions in neural crest development. Bioessays. 2006;28(8):788-798.

41. Gershon TR, Oppenheimer O, Chin SS, Gerald WL. Temporally regulated neural crest transcription factors distinguish neuroectodermal tumors of varying malignancy and differentiation. Neoplasia. 2005;7(6):575-584.

42. Sato A, et al. FGF8 signaling is chemotactic for car- diac neural crest cells. Dev Biol. 2011;354(1):18-30. 43. Khanna C, Jaboin JJ, Drakos E, Tsokos M, Thiele CJ. Biologically relevant orthotopic neuroblastoma xenograft models: primary adrenal tumor growth and spontaneous distant metastasis. In Vivo. 2002; 16(2):77-85.

44. Wesche J, Haglund K, Haugsten EM. Fibroblast growth factors and their receptors in cancer. Biochem J. 2011;437(2):199-213.

45. Ahmad I, Iwata T, Leung HY. Mechanisms of FGFRmediated carcinogenesis. Biochim Biophys Acta. 2012;1823(4):850-860.

46. Kakodkar NC, et al. Sorafenib inhibits neuroblastoma cell proliferation and signaling, blocks angiogenesis, and impairs tumor growth. Pediatr Blood Cancer. 2012;59(4):642-647.

47. Hahn CK, et al. Expression-based screening identifies the combination of histone deacetylase inhibitors and retinoids for neuroblastoma differentiation. Proc Natl Acad Sci U S A. 2008; 105(28):9751-9756.

48. Tsang KS, et al. Detection of micrometastasis of neuroblastoma to bone marrow and tumor dissemination to hematopoietic autografts using flow cytometry and reverse transcriptase-polymerase chain reaction. Cancer. 2003;97(11):2887-2897.

49. Zeltzer PM, Marangos PJ, Evans AE, Schneider SL. Serum neuron-specific enolase in children with neuroblastoma. Relationship to stage and disease course. Cancer. 1986;57(6):1230-1234.

50. Riley RD, et al. A systematic review of molecular and biological tumor markers in neuroblastoma. Clin Cancer Res. 2004;10(1 pt 1):4-12.

51. Kan M, Wang F, Xu J, Crabb JW, Hou J, McKeehan WL. An essential heparin-binding domain in the fibroblast growth factor receptor kinase. Science. 1993;259(5103):1918-1921.

52. Bolstad BM, Irizarry RA, Astrand M, Speed TP. A comparison of normalization methods for high density oligonucleotide array data based on variance and bias. Bioinformatics. 2003; 19(2):185-193.

53. Irizarry RA, Bolstad BM, Collin F, Cope LM, Hobbs B, Speed TP. Summaries of Affymetrix GeneChip probe level data. Nucleic Acids Res. 2003; 31(4):e15.

54. Pei Y, et al. An animal model of MYC-driven medulloblastoma. Cancer Cell. 2012;21(2):155-167.

55. Johnson WE, Li C, Rabinovic A. Adjusting batch effects in microarray expression data using empirical Bayes methods. Biostatistics. 2007;8(1):118-127.

56. Dong $\mathrm{M}$, et al. The type III TGF-beta receptor suppresses breast cancer progression. J Clin Invest. 2007;117(1):206-217.

57. Turley RS, Finger EC, Hempel N, How T, Fields TA, Blobe GC. The type III transforming growth factor-beta receptor as a novel tumor suppressor gene in prostate cancer. Cancer Res. 2007; 67(3):1090-1098.

58. Boergermann JH, Kopf J, Yu PB, Knaus P. Dorsomorphin and LDN-193189 inhibit BMP-mediated Smad, p38 and Akt signalling in C2C12 cells. Int J Biochem Cell Biol. 2010;42(11):1802-1807.

59. Mythreye K, Blobe GC. The type III TGF-beta receptor regulates epithelial and cancer cell migration through beta-arrestin2-mediated activation of Cdc42. Proc Natl Acad Sci US A. 2009;106(20):8221-8226.

60. Finger EC, Lee NY, You HJ, Blobe GC. Endocytosis of the type III transforming growth factor-beta (TGF-beta) receptor through the clathrin-independent/lipid raft pathway regulates TGF-beta signaling and receptor down-regulation. J Biol Chem. 2008;283(50):34808-34818.

61. Lambert KE, Huang H, Mythreye K, Blobe GC. The type III transforming growth factor-beta receptor inhibits proliferation, migration, and adhesion in human myeloma cells. Mol Biol Cell. 2011;22(9):1463-1472. 
62. Chen W, et al. Beta-arrestin 2 mediates endocytosis of type III TGF-beta receptor and down-regulation of its signaling. Science. 2003;301(5638):1394-1397.

63. Blobe GC, et al. Functional roles for the cytoplasmic domain of the type III transforming growth factor beta receptor in regulating transforming growth factor beta signaling. J Biol Chem. 2001;276(27):24627-24637.

64. Boehm JS, et al. Integrative genomic approaches identify IKBKE as a breast cancer oncogene. Cell. 2007;129(6):1065-1079.

65. Meijering E, Jacob M, Sarria JC, Steiner P, Hirling $\mathrm{H}$, Unser M. Design and validation of a tool for neurite tracing and analysis in fluorescence microscopy images. Cytometry A. 2004;58(2):167-176.

66. Mythreye K, Knelson EH, Gatza CE, Gatza ML, Blobe GC. TbetaRIII/beta-arrestin2 regulates integrin alpha5beta1 trafficking, function, and localization in epithelial cells. Oncogene. 2013; 32(11):1416-1427.

67. Gatza CE, et al. Type III TGF-beta receptor enhances colon cancer cell migration and anchorage-independent growth. Neoplasia. 2011;13(8):758-770.

68. Ochiai $\mathrm{H}$, et al. Bmil is a MYCN target gene that regulates tumorigenesis through repression of KIF1Bbeta and TSLC1 in neuroblastoma. Oncogene. 2010;29(18):2681-2690 